\title{
NON BLOW-UP OF THE 3D EULER EQUATIONS FOR A CLASS OF THREE-DIMENSIONAL INITIAL DATA IN CYLINDRICAL DOMAINS*
}

\author{
A. MAHALOV ${ }^{\dagger}$, B. NICOLAENKO ${ }^{\dagger}$, C. BARDOS $\ddagger$ AND F. GOLSE ${ }^{\ddagger}$
}

Dedicated to Professor George Papanicolaou with admiration

\begin{abstract}
Non blow-up of the 3D incompressible Euler Equations is proven for a class of threedimensional initial data characterized by uniformly large vorticity in bounded cylindrical domains. There are no conditional assumptions on the properties of solutions at later times, nor are the global solutions close to some 2D manifold. The approach of proving regularity is based on investigation of fast singular oscillating limits and nonlinear averaging methods in the context of almost periodic functions. We establish the global regularity of the 3D limit resonant Euler equations without any restriction on the size of $3 \mathrm{D}$ initial data. After establishing strong convergence to the limit resonant equations, we bootstrap this into the regularity on arbitrary large time intervals of the solutions of 3D Euler Equations with weakly aligned uniformly large vorticity at $t=0$.
\end{abstract}

Key words. Euler Equations, Vorticity, Regularity

AMS subject classifications. 35B65, 35Q35, 76B03

1. Introduction and main results. Non blow-up of the 3D incompressible Euler Equations is proven for a class of three-dimensional initial data characterized by uniformly large vorticity in bounded cylindrical domains. There are no conditional assumptions on the properties of solutions at later times, nor are the global solutions close to some 2D manifold. It is well known that fully three-dimensional initial conditions with uniformly large vorticity excite fast Poincaré vorticity waves [3], [5]. Since individual Poincaré wave modes are related to the eigenfunctions of the curl operator, they are exact time-dependent solutions of the full nonlinear 3D Euler equations. Of course, their linear superposition does not preserve this property. In this article we prove regularity on arbitrary long time intervals for fully $3 \mathrm{D}$ initial data which are superposition of 'slow' barotropic fields (two-dimensional velocity fields with three components) and an infinite countable spectrum of 'fast' Poincaré waves; this is achieved with a detailed mathematical analysis of multiscale phenomena which arise in the fully nonlinear interactions between 3D waves and the mean flow (barotropic fields). The techniques involve detailed investigations of fast singular oscillating limits, building on the earlier pioneering work on nonlinear averaging methods by Bogoliubov and Mitropolskii [10] and Bensoussan, Lions and Papanicolaou [8].

We study initial value problem for the three-dimensional Euler equations with initial data characterized by uniformly large vorticity:

$$
\begin{aligned}
& \partial_{t} \mathbf{V}+(\mathbf{V} \cdot \nabla) \mathbf{V}=-\nabla p, \nabla \cdot \mathbf{V}=0, \\
& \left.\mathbf{V}(t, y)\right|_{t=0}=\mathbf{V}(0)=\tilde{\mathbf{V}}_{0}(y)+\frac{\Omega}{2} e_{3} \times y
\end{aligned}
$$

where $y=\left(y_{1}, y_{2}, y_{3}\right), \mathbf{V}(t, y)=\left(V_{1}, V_{2}, V_{3}\right)$ is the velocity field and $p$ is the pressure. In Eqs. (1.1) $e_{3}$ denotes the vertical unit vector and $\Omega$ is a constant parameter. The

\footnotetext{
*Received December 24, 2004; accepted for publication March 8, 2004.
${ }^{\dagger}$ Department of Mathematics and Statistics, Arizona State University, Tempe, AZ 85287-1804, USA.

${ }^{\ddagger}$ U.M.R. of Mathematics, University of Paris-7, 75251 Paris Cedex-05, and Laboratoire J.L. Lions, University of Paris-6, France.
} 
field $\tilde{\mathbf{V}}_{0}(y)$ depends on three variables $y_{1}, y_{2}$ and $y_{3}$. Since $\operatorname{curl}\left(\frac{\Omega}{2} e_{3} \times y\right)=\Omega e_{3}$, the vorticity vector at initial time $t=0$ is

$$
\operatorname{curl} \mathbf{V}(0, y)=\operatorname{curl} \tilde{\mathbf{V}}_{0}(y)+\Omega e_{3},
$$

and the initial vorticity has a large component weakly aligned along $e_{3}$, when $\Omega>>1$. These are fully three-dimensional large initial data with large initial $3 \mathrm{D}$ vortex stretching.

Eqs. (1.1) are studied in cylindrical domains

$$
\mathbf{C}=\left\{\left(y_{1}, y_{2}, y_{3}\right) \in \mathbf{R}^{3}: 0<y_{3}<2 \pi / \alpha, y_{1}^{2}+y_{2}^{2}<R^{2}\right\}
$$

where $\alpha$ and $R$ are positive real numbers. If $h$ is the height of the cylinder, $\alpha=2 \pi / h$. Let

$$
\Gamma=\left\{\left(y_{1}, y_{2}, y_{3}\right) \in \mathbf{R}^{3}: 0<y_{3}<2 \pi / \alpha, y_{1}^{2}+y_{2}^{2}=R^{2}\right\} .
$$

Without loss of generality, we can assume that $R=1$. Eqs. (1.1) are considered with periodic boundary conditions in $y_{3}$

$$
\mathbf{V}\left(y_{1}, y_{2}, y_{3}\right)=\mathbf{V}\left(y_{1}, y_{2}, y_{3}+2 \pi / \alpha\right)
$$

and vanishing normal component of velocity on $\Gamma$

$$
\mathbf{V} \cdot \mathbf{N}=\tilde{\mathbf{V}} \cdot \mathbf{N}=0 \text { on } \Gamma
$$

where $\mathbf{N}$ is the normal vector to $\Gamma$. From the invariance of $3 \mathrm{D}$ Euler equations under the symmetry $y_{3} \rightarrow-y_{3}, V_{1} \rightarrow V_{1}, V_{2} \rightarrow V_{2}, V_{3} \rightarrow-V_{3}$, all results in this paper extend to cylindrical domains bounded by two horizontal plates. Then the boundary conditions in the vertical direction are zero flux on the vertical boundaries (zero vertical velocity on the plates). One only needs to restrict vector fields to be even in $y_{3}$ for $V_{1}, V_{2}$ and odd in $y_{3}$ for $V_{3}$, and double the cylindrical domain to $-h \leq y_{3} \leq+h$.

We choose $\tilde{\mathbf{V}}_{0}(y)$ in $\mathbf{L}_{2}(\mathbf{C})$. We introduce $\tilde{\mathbf{V}}(t, y)$ such that

$$
\mathbf{V}(t, y)=\tilde{\mathbf{V}}(t, y)+\frac{\Omega}{2} e_{3} \times y, \operatorname{curl} \mathbf{V}(t, y)=\operatorname{curl} \tilde{\mathbf{V}}(t, y)+\Omega e_{3}
$$

For the vorticity field $\omega=$ curl $\mathbf{V}$ Eqs. (1.1) become

$$
\begin{aligned}
& \frac{\partial}{\partial t} \omega+\mathbf{V} \cdot \nabla \omega=\omega \cdot \nabla \mathbf{V} \\
& \omega(0, y)=\operatorname{curl} \tilde{\mathbf{V}}_{0}(y)+\Omega e_{3}
\end{aligned}
$$

and the initial condition induces large initial vortex stretching.

We present a simple case of results obtained in our joint work with C. Bardos and F. Golse [6], where the initial value problem is solved in more general functional spaces. We establish regularity for arbitrarily large finite times for the 3D Euler solutions for $\Omega$ large, but finite. Our solutions are not close in any sense to those of the 2D or "quasi 2D" Euler and they are characterized by fast oscillations in the $e_{3}$ direction, together with a large vortex stretching term $\omega(t, y) \cdot \nabla \mathbf{V}(t, y)=$ $\omega_{1} \frac{\partial V_{1}}{\partial y_{1}}+\omega_{2} \frac{\partial V_{2}}{\partial y_{2}}+\omega_{3} \frac{\partial V_{3}}{\partial y_{3}}, t \geq 0$ with leading component $\Omega \frac{\partial}{\partial y_{3}} V_{3}(t, y)>>1$. There are 
no assumptions on oscillations in $y_{1}, y_{2}$ for our solutions (nor for the initial condition $\left.\tilde{\mathbf{V}}_{0}(y)\right)$.

Our approach is entirely based on fast singular oscillating limits of Eqs. (1.9)(1.10), nonlinear averaging and cancellation of oscillations in the nonlinear interactions for the vorticity field for large $\Omega$. This has been developed in [3], [4], [5] and [27] for the cases of periodic lattice domains and the infinite space $\mathbf{R}^{3}$. Through the canonical transformation (1.17)-(1.18) in both the field $\mathbf{V}(t, y)$ and the space coordinate $y=$ $\left(y_{1}, y_{2}, y_{3}\right)$ for every $\Omega$ (not necessary large) we map every solution $\mathbf{V}(t, y)$ of Eqs. (1.1) one-to-one to a solution $\mathbf{U}(t, x), x=\left(x_{1}, x_{2}, x_{3}\right)$ of

$$
\begin{aligned}
\partial_{t} \mathbf{U}+\left(\mathbf{U} \cdot \nabla_{x}\right) \mathbf{U}+\Omega \mathbf{e}_{3} \times \mathbf{U} & =-\nabla_{x}\left(p-\frac{\Omega^{2}}{4}\left|x_{h}\right|^{2}\right), \nabla_{x} \cdot \mathbf{U}=0, \\
\left.\mathbf{U}(t, x)\right|_{t=0} & =\mathbf{U}(0, x)=\tilde{\mathbf{V}}_{0}(x),
\end{aligned}
$$

where $x=y$ at $t=0$ and $x_{h}=\left(x_{1}, x_{2}\right)$. For $\Omega>>1$ the nearly singular initial value problem (1.1)-(1.2) (that is with large initial vorticity and vortex stretching) is mapped into the problem (1.11)-(1.12) with the nearly singular Coriolis operator term restricted to solenoidal fields:

$$
\frac{1}{\epsilon} e_{3} \times \mathbf{U}, \nabla \cdot \mathbf{U}=0, \epsilon=1 / \Omega<<1 .
$$

As detailed in Section 2, the linear part of Eq. (1.11) is the Poincaré-Sobolev nonlocal wave equations ([2], [14], [28], [32]):

$$
\partial_{t} \boldsymbol{\Phi}+\Omega \mathbf{e}_{3} \times \boldsymbol{\Phi}=-\nabla \pi, \nabla \cdot \boldsymbol{\Phi}=0 .
$$

Interactions between the Poincaré waves generated by the quadratic nonlinearity in Eq. (1.11) are ruled by resonance conditions and a small divisor problem in the limit $\Omega \rightarrow \infty$. With nonlinear averaging methods in the context of Banach space valued almost periodic functions we obtain fully 3D limit resonant Euler equations. We establish the global regularity of the latter without any restriction on the size of 3D initial data and bootstrap this into the global regularity of Eqs. (1.11)-(1.12) for $\Omega$ large but finite. Then by the canonical transformation (1.17)-(1.18) of the field $\mathbf{V}$ (which is an isometry on curl-based generalizations of Sobolev spaces) we establish the long-time regularity of Eqs. (1.1)-(1.2) for large finite $\Omega$, on arbitrarily finite large time intervals.

Our results crucially use the algebra of the curl operator with boundary conditions, for the fast singular oscillating limits of $\tilde{\omega}=\operatorname{curl} \mathbf{U}(t, x)$ :

$$
\partial_{t} \tilde{\omega}+\mathbf{U} \cdot \nabla \tilde{\omega}=\tilde{\omega} \cdot \nabla \mathbf{U}+\Omega \frac{\partial}{\partial x_{3}} \mathbf{U} .
$$

For this we rely on deep properties of $\mathrm{curl}^{-1}$, extending the early pioneering results of O.A. Ladyzhenskaya, V.A. Solonnikov and co-workers which were obtained in the context of Maxwell's equations and magneto-hydrodynamics ([21], [24], [12], [13], [33]). There are three foremost issues with the analysis of (1.1)-(1.2), (1.11)-(1.15) for large parameter $\Omega$. First, the nature of their fast singular oscillating limit equations as $\Omega \rightarrow+\infty$ and the global regularity of their solutions (3D resonant limit Euler equations). Second, the strong convergence of solutions of (1.11)-(1.12) to those of the limit equations; and, finally, bootstrapping from analysis of the first two questions the long-time regularity of solutions of (1.1)-(1.2) for $\Omega$ large but finite. 
We now detail the canonical transformation between the original vector field $\mathbf{V}(t, y)$ and the vector field $\mathbf{U}(t, x)$. Let $\mathbf{J}$ be the matrix such that $\mathbf{J a}=e_{3} \times \mathbf{a}$ for any vector field a. Then

$$
\mathbf{J}=\left(\begin{array}{ccc}
0 & -1 & 0 \\
1 & 0 & 0 \\
0 & 0 & 0
\end{array}\right), \boldsymbol{\Upsilon}(t) \equiv e^{\Omega \mathbf{J} t / 2}=\left(\begin{array}{ccc}
\cos \left(\frac{\Omega t}{2}\right) & -\sin \left(\frac{\Omega t}{2}\right) & 0 \\
\sin \left(\frac{\Omega t}{2}\right) & \cos \left(\frac{\Omega t}{2}\right) & 0 \\
0 & 0 & 1
\end{array}\right)
$$

For any fixed parameter $\Omega$ (not necessary large) we introduce the following fundamental transformation:

$$
\mathbf{V}(t, y)=e^{+\Omega \mathbf{J} t / 2} \mathbf{U}\left(t, e^{-\Omega \mathbf{J} t / 2} y\right)+\frac{\Omega}{2} \mathbf{J} y, x=e^{-\Omega \mathbf{J} t / 2} y .
$$

The transformation (1.17) is invertible:

$$
\mathbf{U}(t, x)=e^{-\Omega \mathbf{J} t / 2} \mathbf{V}\left(t, e^{+\Omega \mathbf{J} t / 2} x\right)-\frac{\Omega}{2} \mathbf{J} x, y=e^{+\Omega \mathbf{J} t / 2} x .
$$

The transformations (1.17)-(1.18) establish one-to-one correspondence between solenoidal vector fields $\mathbf{V}(t, y)$ and $\mathbf{U}(t, x)$. We note that for $t=0 x=y$ and therefore $\tilde{\mathbf{V}}_{0}(y)=\tilde{\mathbf{V}}_{0}(x)$. Let $x=\left(x_{h}, x_{3}\right)$ where $x_{h}=\left(x_{1}, x_{2}\right),\left|x_{h}\right|^{2}=x_{1}^{2}+x_{2}^{2}$ and similarly for $y$. We have:

LEMMA 1.1. The following identities hold for the vector fields $\mathbf{V}(t, y)$ and $\mathbf{U}(t, x)$ and pressure $p$ :

1. $\nabla_{y} \cdot \mathbf{V}(t, y)=\nabla_{x} \cdot \mathbf{U}(t, x)$.

2. $\nabla_{y} p=\mathbf{\Upsilon}(t) \nabla_{x} p$.

3. $\operatorname{curl}_{y} \mathbf{V}(t, y)=\mathbf{\Upsilon}(t) \operatorname{curl}_{x} \mathbf{U}(t, x)+\Omega e_{3}, \quad \operatorname{curl}_{y}^{2} \mathbf{V}(t, y)=\mathbf{\Upsilon}(t) \operatorname{curl}_{x}^{2} \mathbf{U}(t, x)$.

4. $\frac{D}{D t} \mathbf{V}(t, y)=\mathbf{\Upsilon}(t)\left(\frac{D}{D t} \mathbf{U}(t, x)+\Omega \mathbf{J U}-\frac{\Omega^{2}}{2} x_{h}\right)$ where $\frac{D}{D t}$ are the corresponding Lagrangian derivatives, $\mathbf{J U}=e_{3} \times \mathbf{U}$.

Lemma 1.1 establishes that the transformation (1.17)-(1.18) is canonical for (1.1)(1.2). From the property 1 of Lemma 1.1 it follows that $\nabla_{x} \cdot \mathbf{U}(t, x)=0$ since $\nabla_{y} \cdot \mathbf{V}(t, y)=0$. Now using $2-4$ in the above Lemma 1.1 and the fact that $\mathbf{\Upsilon}(t)$ is unitary we can express each term in (1.1) in $x$ and $t$ variables to obtain the equations for $\mathbf{U}(t, x)$ (1.11)-(1.15). Under the transformation (1.17)-(1.18) Eqs. (1.1)-(1.2) turn into Euler system (1.11)-(1.12) with an additional Coriolis term $\Omega e_{3} \times \mathbf{U}$ and modified initial data and pressure. The systems Eqs. (1.1)-(1.2) and (1.11)-(1.12) are equivalent for every $\Omega$ (not necessary large) and the pair of transformations (1.17)-(1.18) establishes one-to-one correspondence between their fully three-dimensional solutions. The canonical transformation (1.17)-(1.18) preserves the boundary conditions (1.7) which are transformed into

$$
\mathbf{U} \cdot \mathbf{N}=0 \text {, on } \Gamma \text {. }
$$

Using elementary identities $(\mathbf{U} \cdot \nabla) \mathbf{U}=\operatorname{curl} \mathbf{U} \times \mathbf{U}+\nabla\left(\frac{\mid \mathbf{U}^{2}}{2}\right)$ on divergence free vector fields, Eqs. (1.11) can be rewritten in the form

$$
\begin{gathered}
\partial_{t} \mathbf{U}+\left(\operatorname{curl} \mathbf{U}+\Omega e_{3}\right) \times \mathbf{U}=-\nabla\left(p-\frac{\Omega^{2}}{4}\left|x_{h}\right|^{2}+\frac{|\mathbf{U}|^{2}}{2}\right), \\
\nabla \cdot \mathbf{U}=0,\left.\mathbf{U}(t, x)\right|_{t=0}=\mathbf{U}(0)=\tilde{\mathbf{V}}_{0}(x) .
\end{gathered}
$$


For large $\Omega$ the initial value condition (1.2) can be interpreted as weak alignment of the initial vorticity at $t=0$; in the distributional sense, for every test function $\phi(y) \in C_{0}^{\infty}\left(\mathbf{R}^{3}\right)$ we have:

$$
\begin{aligned}
\left|<\operatorname{curl} \mathbf{V}(0, y) / \Omega-e_{3}, \phi(y)>\right| & \left.=\mid<\mathbf{V}(0, y) / \Omega-\frac{1}{2} e_{3} \times y, \operatorname{curl} \phi(y) \nmid 1,22\right) \\
& =\frac{1}{\Omega}|<\mathbf{U}(0, x), \operatorname{curl} \phi(x)>|,
\end{aligned}
$$

with $\Omega \geq \Omega_{1}\left(\Omega_{1}\right.$ is defined in Theorem $\left.1.2, \Omega_{1}>>1\right)$.

The fast singular oscillating limits of Eqs. (1.20)-(1.21) are investigated as $\Omega \rightarrow$ $+\infty$, after further transformation of Eqs. (1.20)-(1.21) with the Poincaré propagator. The latter is the unitary group solution $\mathbf{E}(-\Omega t) \mathbf{\Phi}(0)=\mathbf{\Phi}(t)$ in $L_{2}(\mathbf{C})(\mathbf{E}(0)=\mathbf{I d}$ is the identity) to the linear Poincaré wave problem ([28], [14], [32]):

$$
\partial_{t} \mathbf{\Phi}+\Omega \mathbf{J} \Phi=-\nabla \pi, \quad \nabla \cdot \boldsymbol{\Phi}=0,
$$

or, equivalently,

$$
\partial_{t} \mathbf{\Phi}+\Omega \mathbf{P J P} \boldsymbol{\Phi}=0
$$

where $\mathbf{P}$ is the Leray projection on divergence free vector fields; the solutions $\mathbf{E}(-\Omega t) \mathbf{\Phi}(0)$ are called Poincaré waves. Eqs. (1.23) give rise to a unitary group of transformations $\mathbf{E}(-\Omega t)$ on a space of square-integrable divergence-free vector fields $L_{2}(\mathbf{C})$. The spectrum of the generator of the group of motions, that is the spectrum of the skew-hermitian zero order pseudo-differential operator PJP is $[-i, i]$. In the case of cylindrical domains considered in this paper the eigenvalues (point spectrum) of the operator PJP are dense in $[-i, i]$. The operator PJP has norm one. Since PJP is bounded on $L_{2}(\mathbf{C})$, the solutions to (1.24) with initial condition $\boldsymbol{\Phi}(0)$ is given by

$$
\mathbf{\Phi}(t)=\mathbf{E}(-\Omega t) \mathbf{\Phi}(0)=\sum_{j=0}^{+\infty} \frac{(-\Omega t)^{j}}{j !}(-\mathbf{P J P})^{j} \mathbf{\Phi}(0) .
$$

Applying to Eqs. (1.11)-(1.12) (equivalently, Eqs. (1.20)-(1.21)) the Leray projection $\mathbf{P}$ onto divergence free vector fields, we obtain for $\mathbf{U}=\mathbf{P} \mathbf{U}$

$$
\begin{aligned}
\partial_{t} \mathbf{U}+\Omega \mathbf{P J P} \mathbf{U} & =\mathbf{B}(\mathbf{U}, \mathbf{U}) \\
\left.\mathbf{U}\right|_{t=0} & =\mathbf{U}(0)=\tilde{\mathbf{V}}_{0}
\end{aligned}
$$

where

$$
\mathbf{B}(\mathbf{U}, \mathbf{U})=-\mathbf{P}(\mathbf{U} \cdot \nabla \mathbf{U})=\mathbf{P}(\mathbf{U} \times \operatorname{curl} \mathbf{U}) .
$$

The proofs of regularity rely on the analysis of the dispersion relations for Poincaré waves [28], [14] (solutions to Eqs. (1.23)-(1.24)). The resonance condition for the interactions generated by the Euler quadratic nonlinearity in the limit $\Omega \rightarrow+\infty$ takes the form (see [3] and Sections 2, 3 below):

$$
\pm \frac{k_{3}}{\sqrt{\frac{\beta\left(k_{1}, k_{2}, k_{3}\right)^{2}}{\alpha^{2}}+k_{3}^{2}}} \pm \frac{m_{3}}{\sqrt{\frac{\beta\left(m_{1}, m_{2}, m_{3}\right)^{2}}{\alpha^{2}}+m_{3}^{2}}} \pm \frac{n_{3}}{\sqrt{\frac{\beta\left(n_{1}, n_{2}, n_{3}\right)^{2}}{\alpha^{2}}+n_{3}^{2}}}=0
$$


with the convolution conditions $n_{3}=k_{3}+m_{3}, n_{2}=k_{2}+m_{2}$. Here $m=\left(m_{1}, m_{2}, m_{3}\right)$ are three-dimensional wave vectors. The integers $m_{1}, m_{2}$ and $m_{3}$ are for the radial, azimuthal and axial directions, respectively. Similarly, for $k$ and $n$. Eqs. (1.28) are trivially satisfied for $k_{3}=m_{3}=n_{3}=0$ which correspond to pure two-dimensional interactions (dependence on $x_{1}, x_{2}$ and no dependence on $x_{3}$ in physical space). The nonlinear interactions with $k_{3} m_{3} n_{3}=0, k_{3}^{2}+m_{3}^{2}+n_{3}^{2} \neq 0$ correspond to two-wave resonances and the interactions with $k_{3} m_{3} n_{3} \neq 0$ correspond to strict three-wave resonances. The quantities $\beta$ are related to zeros of certain expressions involving Bessel functions (see Eq. (2.30)).

We outline the structure of the fast oscillating limit equations obtained from Eqs. (1.26) in the limit $\Omega \rightarrow+\infty$ :

$$
\begin{aligned}
\partial_{t} \mathbf{w} & =\tilde{\mathbf{B}}(\mathbf{w}, \mathbf{w}), \\
\left.\mathbf{w}\right|_{t=0} & =\mathbf{w}(0)=\mathbf{U}(0)=\tilde{\mathbf{V}}_{0} .
\end{aligned}
$$

Details are given in Sections 2 and 3.

We denote the orthogonal decomposition $\mathbf{w}=\overline{\mathbf{w}}+\mathbf{w}^{\perp}$ where $\overline{\mathbf{w}}\left(t, x_{1}, x_{2}\right)$ is the barotropic projection (vertical averaging),

$$
\overline{\mathbf{w}}\left(t, x_{1}, x_{2}\right)=\frac{1}{2 \pi \alpha} \int_{0}^{2 \pi / \alpha} \mathbf{w}\left(t, x_{1}, x_{2}, x_{3}\right) d x_{3}
$$

and the orthogonal field $\mathbf{w}^{\perp}\left(t, x_{1}, x_{2}, x_{3}\right)$ verifies $\overline{\mathbf{w}}^{\perp}=0$. Then

$$
\mathbf{w}=\overline{\mathbf{w}}+\mathbf{w}^{\perp} \text {. }
$$

Eqs. (1.29) conserve both energy and helicity. These equations are genuinely threedimensional since they include all $3 \mathrm{D}$ modes but with wave-number interactions restricted in $\tilde{\mathbf{B}}(\mathbf{w}, \mathbf{w})$. We have (see below in Sections 2 and 3 )

$$
\overline{\tilde{\mathbf{B}}(\mathbf{w}, \mathbf{w})}=\tilde{\mathbf{B}}(\overline{\mathbf{w}}, \overline{\mathbf{w}})
$$

implying

$$
\tilde{\mathbf{B}}(\mathbf{w}, \mathbf{w})=\mathbf{B}_{\mathrm{III}}\left(\mathbf{w}^{\perp}, \mathbf{w}^{\perp}\right)+\mathbf{B}_{\mathrm{II}}\left(\overline{\mathbf{w}}, \mathbf{w}^{\perp}\right)+\mathbf{B}_{2 D}(\overline{\mathbf{w}}, \overline{\mathbf{w}})
$$

where $\mathbf{B}_{2 D}$ corresponds to pure $2 \mathrm{D}$ interactions $\left(k_{3}=m_{3}=n_{3}=0\right), \mathbf{B}_{\text {II }}$ is the 'catalytic' operator $\left(k_{3}=0, m_{3} n_{3} \neq 0\right.$ or $\left.m_{3}=0, k_{3} n_{3} \neq 0\right)$. The above implies $k_{3} m_{3} n_{3} \neq 0$ for interactions given by $\mathbf{B}_{\mathrm{III}}$. Such interactions are called strict 3 -wave interactions.

Since $\overline{\mathbf{B}}(\mathbf{w}, \mathbf{w})=\tilde{\mathbf{B}}(\overline{\mathbf{w}}, \overline{\mathbf{w}})$ the solutions $\mathbf{w}\left(t, x_{1}, x_{2}, x_{3}\right)=\left(w_{1}, w_{2}, w_{3}\right)$ of the limit equations (1.29) split into an equation for $\overline{\mathbf{w}}\left(t, x_{1}, x_{2}\right)$ which decouples and an equation for $\mathbf{w}^{\perp}\left(t, x_{1}, x_{2}, x_{3}\right)$ with its coefficients depending on $\overline{\mathbf{w}}$. The field $\overline{\mathbf{w}}\left(t, x_{1}, x_{2}\right)$ satisfies the 2D-3C Euler equations (three components and dependence on two variables $\left.x_{1}, x_{2}\right)$. Specifically,

$$
\begin{aligned}
\partial_{t} \overline{\mathbf{w}}+(\overline{\mathbf{w}} \cdot \nabla) \overline{\mathbf{w}} & =-\nabla_{h} \bar{q}, \nabla_{h} \cdot \overline{\mathbf{w}}=0 \\
\left.\overline{\mathbf{w}}\right|_{t=0} & =\overline{\mathbf{w}}(0)=\overline{\mathbf{U}}(0)
\end{aligned}
$$

where $\nabla_{h}$ denotes the gradient in horizontal variables $x_{1}, x_{2}$. The component $\mathbf{w}^{\perp}\left(t, x_{1}, x_{2}, x_{3}\right)$ (orthogonal to $\overline{\mathbf{w}}$, that is with zero vertical average) satisfies limit equations

$$
\begin{aligned}
\partial_{t} \mathbf{w}^{\perp} & =\mathbf{B}_{\text {II }}\left(\overline{\mathbf{w}}, \mathbf{w}^{\perp}\right)+\mathbf{B}_{\mathrm{III}}\left(\mathbf{w}^{\perp}, \mathbf{w}^{\perp}\right) \\
\left.\mathbf{w}^{\perp}\right|_{t=0} & =\mathbf{w}^{\perp}(0)=\mathbf{U}^{\perp}(0)=\mathbf{U}(0)-\overline{\mathbf{U}}(0) .
\end{aligned}
$$


For $\overline{\mathbf{w}}\left(t, x_{1}, x_{2}\right)$ we have the usual conservation laws and global existence theorems for $2 \mathrm{D}$ Euler ([35], [36]).

For the generic case of no strict 3 wave resonances $\mathbf{B}_{\text {III }}=0$. In this case we have global regularity of the limit resonant equations and long time regularity of the $3 \mathrm{D}$ Euler equations (1.11)-(1.12) for $\Omega$ large. The set of parameters $\alpha$ where $\mathbf{B}_{\text {III }}=0$ has full Lebesgue measure. In such cases, global regularity of the limit resonant equations and long time regularity of the 3D Euler equations (1.11)-(1.12) is proven using the new 3D conservation laws for $\mathbf{B}_{\text {II }}$ (see Section 3) and the convergence Theorem 4.6 in section 4. More precisely, $\mathbf{B}_{\mathrm{III}} \neq 0$ for a countable discrete set of parameters $\alpha$. We now state our main existence theorem. For the cylinder, denote by $h$ the height and $R$ the radius. We denote by $\mathbf{H}_{\sigma}^{s}(\mathbf{C})$ the usual Sobolev spaces of solenoidal vector fields in the cylinder $\mathbf{C}, s \geq 0$.

THEOREM 1.2. Consider the initial value problem for the 3D Euler equations (1.1)-(1.2) with curl $\mathbf{V}(0, y)=\Omega e_{3}+\operatorname{curl} \tilde{\mathbf{V}}_{0}(y)$ and $\tilde{\mathbf{V}}_{0}(y) \in \mathbf{H}_{\sigma}^{s}(\mathbf{C}), s \geq 4$. Let $\operatorname{curl}^{j} \tilde{\mathbf{V}}_{0}(y) \cdot \mathbf{N}=0$ on $\Gamma, 0 \leq j \leq s$. Let $\left\|\tilde{\mathbf{V}}_{0}\right\|_{\mathbf{H}_{\sigma}^{s}(\mathbf{C})} \leq M_{s}$. Let $h / R \notin \mathcal{K}^{*}$ where $\mathcal{K}^{*}$ is a countable discrete set. Let $T_{m}>0$ fixed, arbitrary large. Then there exists $\Omega_{1}\left(h / R, M_{s}, T_{m}\right)$ such that for every fixed $\Omega \geq \Omega_{1}$ there exists a unique regular solution of Eqs. (1.1)-(1.2) for $0 \leq t<T_{m}$ :

$$
\|\tilde{\mathbf{V}}(t, y)\|_{\mathbf{H}_{\sigma}^{s}(\mathbf{C})} \leq \tilde{M}_{s}\left(h / R, M_{s}, T_{m}\right) .
$$

Moreover, curl $^{j} \mathbf{V}(t, y) \cdot \mathbf{N}=0$ on $\Gamma, 0 \leq j \leq s$. For $M_{s}$ fixed, $T_{m} \rightarrow+\infty$ as $1 / \Omega_{1} \rightarrow 0$. Alternatively, we can take arbitrary large but bounded sets of initial data: $M_{s} \rightarrow+\infty$ if $1 / \Omega_{1} \rightarrow 0, T_{m}$ fixed.

The above theorem establishes a class of genuinely 3D solutions of Euler equations which are regular on long time intervals even though the initial vorticity and the vortex stretching term are large.

2. Poincare-Sobolev equations in cylindrical domains. In this section we consider the eigenvalue problem for the Poincaré-Sobolev equations in the cylinder $\mathbf{C}$ $([2],[28],[14],[32])$ :

$$
\partial_{t} \mathbf{\Phi}+\Omega \mathbf{P J P} \boldsymbol{\Phi}=0, \quad \nabla \cdot \boldsymbol{\Phi}=0
$$

The operator PJP is skew-symmetric with respect to the $\mathbf{L}_{2}$ inner product. From the fundamental identity

$$
\operatorname{curl} \mathbf{P J P}=-\frac{\partial}{\partial x_{3}} \mathbf{P},
$$

the Poincaré problem is equivalent to the nonlocal wave operator (the PoincaréSobolev equation), [32] and [2]:

$$
\frac{\partial^{2}}{\partial t^{2}} \operatorname{curl}^{2} \Phi-\Omega^{2} \frac{\partial^{2}}{\partial x_{3}^{2}} \mathbf{P} \Phi=0 ;
$$

its properties have been extensively investigated by the school of Sobolev (see references in [2], [20], [29]) for various domain geometries.

Theorem 2.1. ([20]). PJP is a bounded skew-adjoint zero-order nonlocal operator with a dense spectrum on $[-i,+i]$. 
This spectrum can be purely continuous on $[-i,+i]$ in the case of resonant domains which are ergodically filled by the characteristics of Eqs. (2.3) ([2]). The situation is simpler in periodic domains $\mathbf{T}^{3}$, where $\mathbf{P J P}$ does commute with the curl operator, hence with $\mathrm{curl}^{2 \alpha}=(-\Delta)^{\alpha}$ on solenoidal fields, and $\mathbf{E}(-\Omega t)=\exp (-\Omega \mathbf{P J P} t)$ preserves all Sobolev norms $([3],[4])$.

In the cylindrical domains with boundary conditions ((1.7), (1.19)), the structure of PJP and $\mathbf{E}(-\Omega t)$ is much more complex, as curl does not commute with the operator PJP (but does commute with $\mathbf{P}$ ), whereas the operators $\nabla$ and $-\Delta$ do not commute with $\mathbf{P}$. The Helmholtz projection $\mathbf{U} \rightarrow \mathbf{P U}$ is such that (i) $\operatorname{div} \mathbf{P} \mathbf{U}=0$ and (ii) $\mathbf{P U} \cdot \mathbf{N}=0$ on $\boldsymbol{\Gamma}$. The Weyl-Helmholtz decomposition theorem for $\mathbf{L}_{2}(D)$ where $D$ is a bounded domain, now involves harmonic distributions:

TheOREM 2.2. Every vector field $\mathbf{U} \in \mathbf{L}_{2}(D)$ admits a unique decomposition:

$$
\mathbf{U}=\mathbf{P} \mathbf{U}+\nabla \pi_{H}+\nabla \pi_{0}
$$

where $\nabla \pi_{H}$ and $\nabla \pi_{0} \in \mathbf{L}_{2}(D)$ and

$$
\begin{gathered}
\Delta \pi_{0}=\operatorname{div} \mathbf{U}, \pi_{0}=0 \text { on } \partial D \\
\Delta \pi_{H}=0, \frac{\partial \pi_{H}}{\partial N}=\left(\mathbf{U}-\nabla \pi_{0}\right) \cdot \mathbf{N} \text { on } \partial D, \text { in } \mathbf{H}^{-1 / 2}(\partial D) .
\end{gathered}
$$

Note that the set of harmonic distributions such that $\frac{\partial \pi_{H}}{\partial N}=0$ on $\partial D$, reduces to $\{0\}$, hence the uniqueness of $\mathbf{P U}$.

To construct the eigenfunctions and eigenvalues of PJP and $\mathbf{E}(-\Omega t)$, we need to invert curl in the Poincaré-Sobolev equation (2.3) subject to the boundary condition (1.19). This is where the potential theoretical result on curl inversion by O.A. Ladyzhenskaya and V. A. Solonnikov are needed in an essential way ([21], [12], [13], [24]). V.A. Solonnikov in [33] has further demonstrated that in bounded geometries the curl operator is an overdetermined elliptic system on solenoidal fields and does not admit any simple maximal self-adjoint extension. Nevertheless, two different potential theoretic inverses can be constructed for curl with different domains and ranges.

Recall the lemma for integration by parts for the curl operator

Lemma 2.3. For $\mathbf{U}, \mathbf{V} \in \mathbf{H}^{1}(D)$

$$
\int_{D} \operatorname{curl} \mathbf{U} \cdot \mathbf{V} d x=\int_{D} \mathbf{U} \cdot \operatorname{curl} \mathbf{V} d x+\int_{\partial D}(\mathbf{N} \times \mathbf{U} \cdot \mathbf{V}) d S,
$$

where the determinant in the boundary integral is taken in the sense $\mathbf{N} \times \mathbf{U}$ and $\mathbf{N} \times \mathbf{V}$ $\in \mathbf{H}^{1 / 2}(\partial D)$, and $\mathbf{U}, \mathbf{V} \in \mathbf{H}^{1 / 2}(\partial D)$ ([12], [17]).

Lemma 2.4. ([24]). For $\mathbf{U}, \mathbf{V} \in \mathbf{J}^{0} \cap \mathbf{J}_{1}$ and such that $\operatorname{curl} \mathbf{U} \cdot \mathbf{N}=\operatorname{curl} \mathbf{V} \cdot \mathbf{N}=0$ on $\partial D$, the operator curl is symmetric:

$$
\int_{D} \operatorname{curl} \mathbf{U} \cdot \mathbf{V} d x=\int_{D} \mathbf{U} \cdot \operatorname{curl} \mathbf{V} d x
$$

To briefly review the results in [21], [24], [12], [13] we introduce the notations of Ladyzhenskaya, where $D$ is a bounded domain with boundary $\partial D$ :

$$
\begin{aligned}
& \mathbf{J}=C \operatorname{los}\left\{\mathbf{U} \in C^{\infty}(\bar{D}), \operatorname{div} \mathbf{U}=0\right\} \text { in }\|\cdot\|_{\mathbf{L}_{2}}, \mathbf{J} \equiv \mathbf{H}_{\sigma}^{0} \\
& \mathbf{J}_{1}=\left\{\mathbf{U} \in \mathbf{H}^{1}(D), \operatorname{div} \mathbf{U}=0\right\} \equiv \mathbf{H}_{\sigma}^{1}(D) ; \\
& \mathbf{J}^{0}=\operatorname{Clos}\left\{\mathbf{U} \in C^{\infty}(\bar{D}), \operatorname{div} \mathbf{U}=0, \mathbf{U} \cdot \mathbf{N}=0 \text { on } \partial D\right\} \text { in }\|\cdot\|_{\mathbf{L}_{2}} \\
& \mathbf{J}_{1, \tau}^{0}=\operatorname{Clos}\left\{\mathbf{U} \in C^{\infty}(\bar{D}), \operatorname{div} \mathbf{U}=0, \mathbf{U} \times \mathbf{N}=0 \text { on } \partial D\right\} \text { in }\|\cdot\|_{\mathbf{H}^{1}(D)} .
\end{aligned}
$$


Theorem 2.5. $\mathbf{J}_{1, \tau}^{0}(D)$ is dense in $\mathbf{J}(D)$.

Theorem 2.6. For every $\mathbf{V} \in \mathbf{J}^{0}(D)$, there exists a unique $\Psi \in \mathbf{J}_{1, \tau}^{0}(D)$ such that $\mathbf{V}=\operatorname{curl} \Psi$ and for some $C_{1}, C_{2}>0$ :

$$
C_{1}\|\mathbf{V}\|_{\mathbf{L}_{2}} \leq\|\Psi\|_{\mathbf{H}^{1}} \leq C_{2}\|\mathbf{V}\|_{\mathbf{L}_{2}}
$$

Theorem 2.7. For every $\mathbf{W} \in \mathbf{J}(D)$ there exists a unique $\Phi \in \mathbf{J}^{0}(D) \cap \mathbf{H}^{1}(D)$ such that $\mathbf{W}=\operatorname{curl} \Phi$ and for some $C_{3}, C_{4}>0$ :

$$
C_{3}\|\mathbf{W}\|_{\mathbf{L}_{2}} \leq\|\Phi\|_{\mathbf{H}^{1}} \leq C_{4}\|\mathbf{W}\|_{\mathbf{L}_{2}} .
$$

Theorem 2.6 implies the existence of a bounded operator curl $^{-1}$ with domain $\mathbf{J}^{0}$, range $\mathbf{J}_{1, \tau}^{0}$; and similarly Theorem 2.7 defines curl $^{-1}$ with domain $\mathbf{J}$, range $\mathbf{J}^{0} \cap$ $\mathbf{H}^{1}$. Note that Theorem 2.7 implies the Poincaré inequality $\|\Phi\|_{\mathbf{L}_{2}} \leq C_{4}\|\mathbf{W}\|_{\mathbf{L}_{2}}$. Theorem 2.7 has been rederived by C. Bardos (see discussion in [17]) using nonpotential theoretic methods of K. Friedrichs [18].

TheOrem 2.8. ([24], [2]). When restricted to the domain:

$$
\mathcal{D}(\text { curl })=\text { curl }^{-1} \mathbf{J}^{0},
$$

where the vector potential curl ${ }^{-1}$ is taken as in Theorem 2.7, the operator curl is self-adjoint, invertible and with a compact inverse in $\mathbf{J}^{0}$.

Proof. Theorem 2.8 is a straightforward corollary of Theorem 2.7, with the remark that $\mathbf{J}^{0}$ is a closed subspace of $\mathbf{J}$. $\mathcal{D}(\mathrm{curl})$ is a closed proper subspace of $\mathbf{J}_{1} \cap \mathbf{J}^{0}$. In fact,

$$
\mathbf{J}^{0} \cap \mathbf{J}_{1}=\operatorname{curl}^{-1} \mathbf{J}^{0} \bigoplus \operatorname{curl}^{-1}\left(\nabla \pi_{H}\right),
$$

where curl $^{-1}$ is again taken in the sense of Theorem 2.7. As curl $^{-1} \mathbf{J}^{0} \subset \mathbf{H}_{\sigma}^{1} \cap \mathbf{J}^{0}$, curl $^{-1}$ is a compact operator on $\mathbf{J}^{0}$. To verify that curl as restricted in (2.15) is self-adjoint, consider (2.7) with $\mathbf{U}, \mathbf{V}$ in $\mathcal{D}($ curl $)$ and $\Psi_{U}, \Psi_{V}$ in $\mathbf{J}_{1, \tau}^{0}$ such that $\Psi_{U}=\operatorname{curl}^{-1}(\operatorname{curl} \mathbf{U}), \Psi_{V}=\operatorname{curl}^{-1}(\operatorname{curl} \mathbf{V})$, the vector potential $\operatorname{curl}^{-1}$ is now taken in the sense of Theorem 2.6. Then $\Psi_{U}=\mathbf{U}+\nabla \pi_{H}(\mathbf{U}), \Psi_{V}=\mathbf{U}+\nabla \pi_{H}(\mathbf{V})$, where $\pi_{H}(\mathbf{U}), \pi_{H}(\mathbf{V})$ are harmonic functions. Now

$$
\int_{D} \operatorname{curl} \mathbf{U} \cdot \mathbf{V} d x=\int_{D} \operatorname{curl} \mathbf{U} \cdot \Psi_{V} d x=\int_{D} \operatorname{curl} \Psi_{U} \cdot \Psi_{V} d x
$$

and, similarly,

$$
\int_{D} \mathbf{U} \cdot \operatorname{curl} \mathbf{V} d x=\int_{D} \Psi_{U} \cdot \operatorname{curl} \Psi_{V} d x
$$

Then (2.7) becomes

$$
\int_{D} \operatorname{curl} \mathbf{U} \cdot \mathbf{V} d x=\int_{D} \mathbf{U} \cdot \operatorname{curl} \mathbf{V} d x+\int_{\partial D}\left(\mathbf{N} \times \Psi_{U} \cdot \Psi_{V}\right) d S,
$$

and the boundary integral on the right hand side is clearly null, as $\Psi_{U} \cdot \Psi_{V}$ are in $\mathbf{J}_{1, \tau}^{0}$. $\square$ 
Theorem 2.8 has been rediscovered several times, from unawareness of [24] and [13]. Note that whereas the eigenfunctions of $\mathrm{curl}$ are complete in $\mathbf{J}^{0}$, they are not complete in $\mathbf{J}^{0} \cap \mathbf{J}_{1}$, only in $\mathcal{D}($ curl $) \subset \mathbf{H}^{1}(D)$.

We now explicit the common eigenfunctions to PJP and curl in the cylinder. In cylindrical coordinates $(r, \phi, z)$ we have $\boldsymbol{\Phi}=\left(\Phi_{r}, \Phi_{\phi}, \Phi_{z}\right)$ and Eqs. (2.1) take the form

$$
\begin{array}{r}
\partial_{t} \Phi_{r}-\Omega \Phi_{\phi}=-\frac{\partial p}{\partial r}, \partial_{t} \Phi_{\phi}+\Omega \Phi_{r}=-\frac{1}{r} \frac{\partial p}{\partial \phi}, \partial_{t} \Phi_{z}=-\frac{\partial p}{\partial z} \\
\frac{1}{r} \frac{\partial}{\partial r}\left(r \Phi_{r}\right)+\frac{1}{r} \frac{\partial \Phi_{\phi}}{\partial \phi}+\frac{\partial \Phi_{z}}{\partial z}=0 .
\end{array}
$$

The vector field $\boldsymbol{\Phi}$ is $2 \pi / \alpha$ periodic in $z$ and it satisfies $\left.\Phi_{r}\right|_{r=R}=0$ on $\Gamma$.

Applying curl operator to Eqs. (1.23) and using divergence free condition, we obtain

$$
\partial_{t} \operatorname{curl} \boldsymbol{\Phi}=\Omega \partial_{z} \boldsymbol{\Phi} .
$$

From Eqs. (2.22) we obtain Poincaré-Sobolev equations

$$
\frac{\partial^{2}}{\partial t^{2}}\left(\operatorname{curl}^{2} \boldsymbol{\Phi}\right)-\Omega^{2} \frac{\partial^{2}}{\partial z^{2}}(\boldsymbol{\Phi})=0 .
$$

Eqs. (2.23) is a system of equations for three components of $\boldsymbol{\Phi}$. For the vertical component $\Phi_{z}$ we have the following scalar equation

$$
\frac{\partial^{2}}{\partial t^{2}} \Delta \Phi_{z}+\Omega^{2} \frac{\partial^{2}}{\partial z^{2}} \Phi_{z}=0
$$

We look for normal modes in the form

$$
e^{i\left(\Omega \sigma t+m_{2} \phi+m_{3} \alpha z\right)} \hat{\boldsymbol{\Phi}}(r) .
$$

Recall that without loss of generality $R=1$. Eqs. (2.24) imply

$$
\frac{d^{2}}{d r^{2}} \hat{\Phi}_{z}+\frac{1}{r} \frac{d}{d r} \hat{\Phi}_{z}+\left(\beta^{2}-\frac{m_{2}^{2}}{r^{2}}\right) \hat{\Phi}_{z}=0
$$

where

$$
\beta^{2}=m_{3}^{2} \alpha^{2}\left(\frac{1}{\sigma^{2}}-1\right) \text { or equivalently } \sigma^{2}=\frac{m_{3}^{2} \alpha^{2}}{\beta^{2}+m_{3}^{2} \alpha^{2}} .
$$

From the boundary condition $\left.\Phi_{r}\right|_{r=1}=0$ and Eqs. (2.20) we obtain

$$
\frac{d}{d r} \hat{\Phi}_{z}+\frac{m_{2}}{\sigma r} \hat{\Phi}_{z}=0 \text { at } \mathrm{r}=1
$$

Eqs. (2.26), (2.28) is a Sturm-Liouville eigenvalue problem.

From Eqs. (2.26) we have

$$
\hat{\Phi}_{z}(r)=J_{m_{2}}(\beta r),
$$

where $\mathbf{J}_{m_{2}}(\cdot), m_{2}=0,1,2, \ldots$, are Bessel functions of the first kind; therefore, Eqs. (2.28) imply

$$
\beta J_{m_{2}}^{\prime}(\beta) \pm m_{2} J_{m_{2}}(\beta) \sqrt{\frac{\beta^{2}}{m_{3}^{2} \alpha^{2}}+1}=0
$$


For fixed integers $m_{2}$ (azimuthal wavenumber) and $m_{3}$ (vertical direction) we solve Eqs. (2.30) to obtain $\beta_{m_{1}}\left(m_{2}, m_{3}\right) ; m_{1}=1,2,3, \ldots$ Eqs. (2.30) have infinitely many solutions. Then Eqs. (2.27) imply

$$
\sigma\left(m_{1}, m_{2}, m_{3}\right)= \pm \frac{m_{3}}{\sqrt{\frac{\beta\left(m_{1}, m_{2}, m_{3}\right)^{2}}{\alpha^{2}}+m_{3}^{2}}} .
$$

Clearly, $i \sigma\left(m_{1}, m_{2}, m_{3}\right)$ are eigenvalues of the skew-hermitian operator PJP. The corresponding eigenvector functions $\boldsymbol{\Phi}_{m_{1} m_{2} m_{3}}=\left(\Phi_{r}, \Phi_{\phi}, \Phi_{z}\right)$ form a complete set in $\mathbf{J}^{0}$. They are independent of $\Omega$ and are explicitly expressed in terms of Bessel functions (for example, see Eq. (2.29) for the radial component $\Phi_{r}$ ). From Eqs. (2.22) and (2.27) it follows that the eigenfunctions $\Phi_{m_{1} m_{2} m_{3}}$ with $m_{3} \neq 0$ satisfy

$$
\begin{aligned}
\operatorname{curl} \Phi_{m_{1} m_{2} m_{3}} & = \pm \sqrt{\beta\left(m_{1}, m_{2}, m_{3}\right)^{2}+\alpha^{2} m_{3}^{2}} \Phi_{m_{1} m_{2} m_{3}} \\
& = \pm \lambda_{m_{1} m_{2} m_{3}} \boldsymbol{\Phi}_{m_{1} m_{2} m_{3}} \\
& = \pm \frac{m_{3} \alpha}{\sigma_{m_{1} m_{2} m_{3}}} \Phi_{m_{1} m_{2} m_{3}} \quad \text { where } \sigma_{m_{1} m_{2} m_{3}}^{2}=\frac{m_{3}^{2} \alpha^{2}}{\beta_{m_{1} m_{2} m_{3}}^{2}+m_{3}^{2} \alpha^{2}}
\end{aligned}
$$

and

$$
\left.(\operatorname{curl} \Phi) \cdot \mathbf{N}\right|_{\Gamma}=0 .
$$

More precisely, from $\operatorname{curl} \mathbf{P J P U}=-\frac{\partial}{\partial z} \mathbf{U}$ (for every solenoidal field $\mathbf{U}$ ), $\Phi_{m_{1} m_{2} m_{3}}$ satisfies

$$
\begin{array}{r}
\operatorname{curl} \Phi_{m_{1} m_{2} m_{3}}=-\frac{\check{m}_{3}}{\sigma} \Phi_{m_{1} m_{2} m_{3}}, \\
\operatorname{curl} \Phi_{m_{1} m_{2} m_{3}}=-\operatorname{sign}\left(m_{3} \sigma\right) \lambda_{m_{1} m_{2} m_{3}} \Phi_{m_{1} m_{2} m_{3}},
\end{array}
$$

where the curl operator is complex valued on the Fourier wavenumbers $m_{2}, m_{3}$ and real valued with respect to the radial variable $r$.

The divergence free eigenvector functions $\Phi_{m_{1} m_{2} m_{3}}=\left(\Phi_{r}, \Phi_{\phi}, \Phi_{z}\right)$ are

$$
\begin{aligned}
\Phi_{r, m_{1} m_{2} m_{3}} & =e^{i\left(m_{2} \phi+m_{3} \alpha z\right)} \frac{i \sigma}{m_{3} \alpha\left(1-\sigma^{2}\right)}\left(\sigma \beta J_{m_{2}}^{\prime}(\beta r)+\frac{m_{2}}{r} J_{m_{2}}(\beta r)\right), \\
\Phi_{\phi, m_{1} m_{2} m_{3}} & =e^{i\left(m_{2} \phi+m_{3} \alpha z\right)} \frac{-\sigma}{m_{3} \alpha\left(1-\sigma^{2}\right)}\left(\beta J_{m_{2}}^{\prime}(\beta r)+\frac{\sigma m_{2}}{r} J_{m_{2}}(\beta r)\right), \\
\Phi_{z, m_{1} m_{2} m_{3}} & =e^{i\left(m_{2} \phi+m_{3} \alpha z\right)} J_{m_{2}}(\beta r) .
\end{aligned}
$$

The eigenspace corresponding to the zero eigenvalue consists of all divergence free vector fields independent of the vertical coordinate $z\left(\sigma=0\right.$ if $m_{3}=0$ in Eqs. (2.31)).

These eigenfunctions are vested with several symmetries, one of them especially useful for the proof of the 'operator splitting' Theorem 3.2, cf. Eqs. (1.34)-(1.35). If

$$
m_{2} \rightarrow-m_{2}, m_{3} \rightarrow-m_{3}, \sigma \rightarrow-\sigma,
$$

then:

$$
\beta\left(m_{1},-m_{2},-m_{3}\right)=\beta\left(m_{1}, m_{2}, m_{3}\right),
$$

with the same indexation $m_{1}$, and:

$$
\Phi^{*}\left(m_{1}, m_{2}, m_{3}\right)=\Phi\left(m_{1},-m_{2},-m_{3}\right) .
$$


Moreover, these two eigenfunctions correspond to the same eigenvalue of curl. Here * denotes complex conjugation. Eqs. (2.36)-(2.38) are easily inferred from (2.28), (2.30), (2.31), (2.32), (2.35) and the above explicit formulas for $\Phi_{m_{1} m_{2} m_{3}}$.

We can easily obtain asymptotic expressions of eigenvalues for large $\beta$. We recall that we have for Bessel functions

$$
\begin{gathered}
J_{l}^{\prime}(\xi)=\frac{l}{\xi} J_{l}(\xi)-J_{l+1}(\xi), \\
J_{l+1}(\xi)=\frac{2 l}{\xi} J_{l}(\xi)-J_{l-1}(\xi), \\
J_{l-1}(\xi)-J_{l+1}(\xi)=2 J_{l}^{\prime}(\xi) \\
J_{l}(\xi) \sim \sqrt{\frac{2}{\pi \xi}} \cos \left(\xi-\frac{\pi}{4}-\frac{l \pi}{2}\right) \text { as } \xi \rightarrow+\infty .
\end{gathered}
$$

From Eqs. (2.30), (2.39)-(2.41) we obtain

$$
\frac{J_{m_{2}+1}(\beta)}{J_{m_{2}}(\beta)}=\frac{m_{2}}{\beta}\left(1 \pm \sqrt{\left.\frac{\beta^{2}}{m_{3}^{2} \alpha^{2}}+1\right)} .\right.
$$

Then from Eqs. (2.43) using asymptotic expression for Bessel functions for large $\beta$ we have

$$
\tan \left(\beta-\frac{\pi}{4}-\frac{m_{2} \pi}{2}\right) \approx \pm \frac{m_{2}}{m_{3} \alpha}
$$

For fixed $m_{2}, m_{3}$ and $\alpha$ Eqs. (2.44) has infinitely many solutions $\beta_{m_{1}}\left(m_{2}, m_{3}, \alpha\right)$, $m_{1}=1,2, \ldots$

In summary, with $\check{n}_{3}=\alpha n_{3}=2 \pi n_{3} / h$ denoting the vertical Fourier wave number along $x_{3}$, we have established that:

Proposition 2.9.

(i) On $[-i, 0) \cup(0,+i]$ the spectrum of $\mathbf{P J P}$ consists of a dense, but countable set of eigenvalues $\pm i \sigma_{n}$, with finite dimensional eigenspaces for each eigenvalue.

(ii) Every eigenvector of $\mathbf{P J P}$ is an eigenvector of curl and vice-versa, with eigenvalues $\pm i \sigma_{n}$ and $\pm \lambda_{n}$ mapped into each other by $\sigma_{n}^{2}=\frac{\check{n}_{3}^{2}}{\lambda_{n}^{2}}$.

(iii) $\operatorname{ker} \mathbf{P J P}=$

$\left\{\mathbf{U} \in \mathbf{J}(\mathbf{C}): \mathbf{U} \equiv \overline{\mathbf{U}}\left(x_{1}, x_{2}\right)=\left(U_{1}\left(x_{1}, x_{2}\right), U_{2}\left(x_{1}, x_{2}\right), U_{3}\left(x_{1}, x_{2}\right)\right)\right\}$.

(iv) On $(k e r \mathbf{P J P})^{\perp}, \mathbf{E}(-\Omega t)=\exp (-\mathbf{P J P} \Omega t)$ is diagonalized in the curleigenvector functions basis, with eigenvalues $\exp \left( \pm i \Omega \sigma_{n} t\right)=\exp \left( \pm i \Omega \frac{\check{n}_{3}}{\lambda_{n}} t\right)$

Completeness of the eigenfunctions of PJP follows from that of the curl eigenfunctions in $\mathbf{J}^{0}$, Eq. (2.11), and every real valued solenoidal field $\mathbf{U}^{\perp}$ satisfying the boundary conditions $(1.7),(1.19)$ and such that $\overline{\mathbf{U}}^{\perp}=0$, can be expanded as:

$$
\mathbf{U}^{\perp}=\sum_{n_{1}>0, n_{2} \geq 0, n_{3}>0}\left(\mathbf{U}_{n_{1}, n_{2}, n_{3}}^{\perp} \Phi_{n_{1}, n_{2}, n_{3}}+\mathbf{U}_{n_{1},-n_{2},-n_{3}}^{\perp} \Phi_{n_{1},-n_{2},-n_{3}}\right),
$$

where $\left(\mathbf{U}_{n_{1} n_{2} n_{3}}^{\perp}\right)^{*}=\mathbf{U}_{n_{1},-n_{2},-n_{3}}^{\perp}$, and $n_{1}$ designates the indexing of $\beta_{n_{1}}\left(n_{2}, n_{3}, \alpha\right)$ for solutions of (2.30), $n_{2}, n_{3}$ the usual azimuthal and vertical wavenumbers. In the next sections, we use the short hand notation $\mathbf{U}_{n}^{\perp}=\mathbf{U}_{n_{1} n_{2} n_{3}}^{\perp}, \Phi_{n}=\Phi_{n_{1} n_{2} n_{3}}$ and for concision's sake, $-n=\left(n_{1},-n_{2},-n_{3}\right), \mathbf{U}_{-n}^{\perp}=\mathbf{U}_{n_{1}-n_{2}-n_{3}}^{\perp}, \Phi_{-n}=\Phi_{n_{1}-n_{2}-n_{3}}$, with the 
understanding that any convolution operator acts only on the Fourier wavenumbers $n_{2}, n_{3}$; for example:

$$
(\mathbf{U} \times \mathbf{V})_{n}=\sum_{m_{1}, m_{2}, m_{3}: m_{2}+k_{2}=n_{2} ; k_{1}, k_{2}, k_{3}: m_{3}+k_{3}=n_{3}}\left(\Phi_{k} \times \Phi_{m}, \Phi_{n}\right) U_{k} V_{m},
$$

for both $\mathbf{U}$ and $\mathbf{V}$ in $(k e r \mathbf{P J P})^{\perp}$.

REMARK 2.10. We note that

$$
\mathbf{V}(t, y)=\frac{\Omega}{2} e_{3} \times y+\exp \left(\frac{\Omega}{2} \mathbf{J} t\right) \Phi_{n}\left(\exp \left(-\frac{\Omega}{2} \mathbf{J} t\right) y\right) \exp \left( \pm i \frac{\check{n}_{3}}{\left|\lambda_{n}\right|} \Omega t\right),
$$

where $\Phi_{n}$ are curl eigenfunctions and $\lambda_{n}$ the corresponding eigenvalues, are exact solutions of the full 3D nonlinear Euler equations. It follows from the fact that the second component in (2.47) is an Arnold-Beltrami-Childress (ABC) flow since $\Phi_{n}$ is a curl eigenfunction; such 3D flows are characterized by colinear velocity and vorticity fields at every point in space and time $([2]),([15])$.

\section{The structure and regularity of fast singular oscillating limit equa-} tions.

3.1. Fast singular oscillating limit equations. We introduce van der Pol transformation by setting in Eqs. (1.11)-(1.20)

$$
\mathbf{U}(t)=\mathbf{E}(-\Omega t) \mathbf{u}(t)
$$

where $\mathbf{u}(t)$ is the "slow envelope" variable also denoted in this paper by Poincaré variable. We note that $\mathbf{E}(-\Omega t)=\exp (-\Omega \mathbf{P} \mathbf{J P} t)$ reduces to the identity operator on any barotropic (vertically averaged) field implying

$$
\overline{\mathbf{U}}=\overline{\mathbf{E}(-\Omega t) \mathbf{u}}=\overline{\mathbf{u}} .
$$

Since $\left.\mathbf{E}(\Omega t)\right|_{t=0}=\mathbf{I d}$

$$
\left.\mathbf{U}\right|_{t=0}=\left.\mathbf{u}\right|_{t=0} .
$$

Eqs. (1.26) written in $\mathbf{u}$ variables have the form ([3])

$$
\begin{aligned}
\partial_{t} \mathbf{u} & =\mathbf{B}(\Omega t, \mathbf{u}, \mathbf{u}), \\
\mathbf{B}(\Omega t, \mathbf{u}, \mathbf{u}) & =\mathbf{E}(\Omega t) \mathbf{B}(\mathbf{E}(-\Omega t) \mathbf{u}, \mathbf{E}(-\Omega t) \mathbf{u})
\end{aligned}
$$

where $\mathbf{B}$ is given by Eqs. (1.27). We decompose

$$
\mathbf{B}(\Omega t, \mathbf{u}, \mathbf{u})=\tilde{\mathbf{B}}(\mathbf{u}, \mathbf{u})+\mathbf{B}^{o s c}(\Omega t, \mathbf{u}, \mathbf{u}) .
$$

Here $\mathbf{B}^{\text {osc }}(\Omega t, \mathbf{u}, \mathbf{u})$ contains all $\Omega$ - dependent terms (that is non-resonant) and $\tilde{\mathbf{B}}(\mathbf{u}, \mathbf{u})$ contains all resonant (that is $\Omega t$ - independent) terms.

The fast singular oscillating limit equations ([3], [4]) are obtained from (3.4) for 'slow' Poincaré variables $\mathbf{w}$ by dropping $\mathbf{B}^{\text {osc }}(\Omega t, \mathbf{u}, \mathbf{u})$ in $(3.5)$ :

$$
\begin{aligned}
\partial_{t} \mathbf{w} & =\tilde{\mathbf{B}}(\mathbf{w}, \mathbf{w}), \\
\left.\mathbf{w}\right|_{t=0} & =\mathbf{w}(0)=\mathbf{U}(0)=\tilde{\mathbf{V}}_{0} .
\end{aligned}
$$


Here the operator $\tilde{\mathbf{B}}$ is defined by (see Lemma 3.1 and section 4 for a rigorous statement)

$$
\tilde{\mathbf{B}}(\mathbf{v}(t), \mathbf{v}(t))=\lim _{\Omega \rightarrow+\infty} \frac{1}{T} \int_{0}^{T} \mathbf{B}(\Omega s, \mathbf{v}(t), \mathbf{v}(t)) d s=\lim _{T \rightarrow+\infty} \frac{1}{T} \int_{0}^{T} \mathbf{B}(\Omega s, \mathbf{v}(t), \mathbf{v}(t)) d s
$$

where arguments $\mathbf{v}$ are $s$-independent functions; limits are taken in the sense of almost periodic functions in $s$ with values in Banach spaces $A P\left(\mathbf{R} ; C^{0}\left([0, T] ; \mathbf{H}_{\nu}^{s}\right)\right)[9],[16]$; see Section 4 for a detailed review and discussion.

The limit resonant operator $\tilde{\mathbf{B}}$ inherits properties of the operator $\mathbf{B}$ :

Lemma 3.1. ([4]): Let $(\mathbf{u}, \mathbf{v}, \mathbf{w}) \in H_{1} \times H_{1} \times H_{1}$. Then

$$
\begin{aligned}
(\tilde{\mathbf{B}}(\mathbf{u}, \mathbf{v}), \mathbf{w}) & =\lim _{\Omega \rightarrow \infty} \frac{1}{T} \int_{0}^{T}(\mathbf{B}(\Omega s, \mathbf{u}, \mathbf{v}), \mathbf{w}) d s \\
& =\lim _{T \rightarrow \infty} \frac{1}{T} \int_{0}^{T}(\mathbf{B}(\Omega s, \mathbf{u}, \mathbf{v}), \mathbf{w}) d s ;
\end{aligned}
$$

in particular, for solenoidal fields $\mathbf{u}, \mathbf{v}$ and $\mathbf{w}$ :

$$
(\tilde{\mathbf{B}}(\mathbf{u}, \mathbf{v}), \mathbf{w})=-(\tilde{\mathbf{B}}(\mathbf{u}, \mathbf{w}), \mathbf{v}) .
$$

From now on we shall restrict the initial data (1.12), (1.21), (1.29), (3.3), (3.7) to the closed (proper) subspace of $\mathbf{J}^{0} \cap \mathbf{H}_{\sigma}^{s}, s \geq 3, s$ integer, defined by (with the potential vector curl $^{-1}$ as in Theorem 2.7):

$$
\mathbf{H}_{\nu}^{s}(\mathbf{C})=\mathbf{J}^{0} \cap \mathbf{H}_{\sigma}^{s} \cap \operatorname{curl}^{-s}\left(\mathbf{J}^{0}\right) .
$$

We will similarly restrict solutions of Eqs. (1.1)-(1.2) to the space $\mathbf{H}_{\nu}^{s}(\mathbf{C})$ (see Theorem 4.5).

Remark 3.1. $\mathbf{v} \in \mathbf{H}_{\nu}^{s}$ is equivalent to $\mathbf{v} \in \mathbf{J}^{0} \cap \mathbf{H}_{\sigma}^{s}$ and curl $^{j} \mathbf{v} \in \mathbf{J}^{0}, 0 \leq j \leq s$. The complement of $\mathbf{H}_{\nu}^{s}$ in $\mathbf{H}_{\sigma}^{s}$ includes functions such as curl ${ }^{-j}\left(\nabla \pi_{H}\right), 1 \leq j \leq s$ and is not dense even in $\mathbf{H}_{\sigma}^{1}$. The case of more general initial conditions and functional spaces for Eqs. (1.1)-(1.2) will be treated in [6].

We can explicit the limit resonant operator $\tilde{\mathbf{B}}$ with the help of the eigenfunctions $\Phi_{n}=\Phi_{n_{1} n_{2} n_{3}}$ of curl and PJP, which form a basis in the space $\mathbf{H}_{\nu}^{s}$; expand

$$
\mathbf{u}=\sum_{n} u_{n} \Phi_{n}=\overline{\mathbf{u}}+\sum_{n} u_{n}^{\perp} \Phi_{n}
$$

and for every $f$ in $\mathbf{J}^{0}$,

$$
\mathbf{E}(\Omega t) f=\bar{f}+\sum_{n} \exp \left(i \Omega \sigma_{n} t\right) f_{n}^{\perp} \Phi_{n} .
$$

From the diagonalization of $\mathbf{E}(-\Omega t)$ and curl operator:

$$
\begin{gathered}
\partial_{t} \mathbf{u}=\sum_{k, m: k_{3}, m_{3} \neq 0} \overline{\mathbf{B}\left(\overline{\mathbf{u}}+\mathbf{u}_{k}^{\perp} \exp \left(-i \sigma_{k} \Omega t\right) \Phi_{k}, \overline{\mathbf{u}}+\mathbf{u}_{m}^{\perp} \exp \left(-i \sigma_{m} \Omega t\right) \Phi_{m}\right)}+ \\
\sum_{n: n_{3} \neq 0} \sum_{k, m: k_{3}, m_{3} \neq 0}\left(\mathbf{B}\left(\overline{\mathbf{u}}+\mathbf{u}_{k}^{\perp} \exp \left(-i \sigma_{k} \Omega t\right) \Phi_{k}, \overline{\mathbf{u}}+\mathbf{u}_{m}^{\perp} \exp \left(-i \sigma_{m} \Omega t\right) \Phi_{m}\right), \Phi_{n}\right) \exp \left(i \sigma_{n} \Omega t\right) \Phi_{n}
\end{gathered}
$$


where $k, m, n$ now index the eigenvalues and eigenfunctions of curl and PJP.

The resonant nonlinear interactions of Poincaré waves with $\mathbf{B}(\mathbf{U}, \mathbf{U})$ are present when the Poincaré frequencies satisfy the relation $\pm \sigma_{k} \pm \sigma_{m} \pm \sigma_{n}=0, k_{3}+m_{3}=n_{3}$, with the resonant set $K$ now defined in terms of vertical wavenumbers $k_{3}, m_{3}, n_{3}$ and eigenvalues $\pm \lambda_{k}, \pm \lambda_{m}, \pm \lambda_{n}$ of curl:

$$
K=\left\{ \pm \frac{k_{3}}{\lambda_{k}} \pm \frac{m_{3}}{\lambda_{m}} \pm \frac{n_{3}}{\lambda_{n}}=0, n_{3}=k_{3}+m_{3}, n_{2}=k_{2}+m_{2}\right\}
$$

Since the eigenvalues of curl are countable, so are the $D_{l}(k, m, n)= \pm \sigma_{k} \pm \sigma_{m} \pm \sigma_{n}$, where $k, m, n$ are now indexing the eigenfunctions and eigenvalues of PJP (and curl). This fully justifies the existence of a mean in the sense of almost periodic functions in the space $A P\left(\mathbf{R} ; C^{0}\left([0, T] ; \mathbf{H}_{\nu}^{s}\right)\right)$ for:

$$
\tilde{\mathbf{B}}(\mathbf{v}(t), \mathbf{v}(t))=\lim _{T \rightarrow+\infty} \frac{1}{T} \int_{0}^{T} \mathbf{E}(\Omega s) \mathbf{B}(\mathbf{E}(-\Omega s) \mathbf{v}(t), \mathbf{E}(-\Omega s) \mathbf{v}(t)) d s
$$

since the frequency spectrum of $\mathbf{E}(\Omega s)$ is countable, following the countability of $D_{l}(k, m, n)$.

Recall that $\overline{\mathbf{w}}$ designates the projection of any $\mathbf{w}$ onto the nullspace of PJP, cf. Proposition 2.9, and $\mathbf{w}^{\perp}$ its projection onto the orthogonal complement of that nullspace (Eqs.(1.30)-(1.31)). We can further explicit the component $\tilde{\mathbf{B}}(\mathbf{w}, \mathbf{w})^{\perp}$ of the limit resonant operator $\tilde{\mathbf{B}}(\mathbf{w}, \mathbf{w})$ :

$$
\tilde{\mathbf{B}}(\mathbf{w}, \mathbf{w})^{\perp}=\mathbf{B}_{\mathrm{II}}\left(\overline{\mathbf{w}}, \mathbf{w}^{\perp}\right)+\mathbf{B}_{\mathrm{III}}\left(\mathbf{w}^{\perp}, \mathbf{w}^{\perp}\right),
$$

where $\mathbf{B}_{\text {II }}$ involves only strict two-wave resonances and $\mathbf{B}_{\text {III }}$ strict three-wave resonances; let:

$$
\mathbf{w}^{\perp}=\sum_{m_{1} m_{2} m_{3}} w_{m_{1} m_{2} m_{3}}^{\perp} \Phi_{m_{1} m_{2} m_{3}}(r, \phi, z)
$$

then

$\mathbf{B}_{\text {II }}\left(\overline{\mathbf{w}}, \mathbf{w}^{\perp}\right)_{n}=\sum_{m, m_{3}=n_{3}, \beta_{m}=\beta_{n}}\left[\left(\operatorname{curl} \overline{\mathbf{w}} \times w_{m}^{\perp} \Phi_{m}, \Phi_{n}\right)+\left(w_{m}^{\perp} \operatorname{curl} \Phi_{m} \times \overline{\mathbf{w}}, \Phi_{n}\right)\right]$,

using the complex form of the curl eigenfunctions $\Phi_{m_{1}, m_{2}, m_{3}} \equiv \Phi_{m}$ in Eqs.(2.29)(2.31), with $\beta_{m} \equiv \beta_{m_{1} m_{2} m_{3}}$. Furthermore,

$$
\mathbf{B}_{\mathrm{III}}\left(\mathbf{w}^{\perp}, \mathbf{w}^{\perp}\right)_{n}=\sum_{k, m, n \in K^{*}}\left(\operatorname{curl} \Phi_{k} \times \Phi_{m}, \Phi_{n}\right) w_{k}^{\perp} w_{m}^{\perp}
$$

here $K^{*} \subset K$ designates the subset of strict 3-waves resonances, $K^{*} \subset\{k, m, n \in$ $\left.K: k_{3} m_{3} n_{3} \neq 0\right\}$. In these formulas, we have explicitly used that the curl operator commutes with the projection operator onto the nullspace of PJP:

$$
\operatorname{curl} \overline{\mathbf{u}}=\overline{\operatorname{curl} \mathbf{u}}, \operatorname{curl} \mathbf{u}^{\perp}=(\operatorname{curl} \mathbf{u})^{\perp}, \operatorname{curl} \mathbf{u}=\operatorname{curl} \overline{\mathbf{u}}+\operatorname{curl} \mathbf{u}^{\perp} .
$$

These properties are important in proving that the nonlinear operator $\tilde{\mathbf{B}}$ commutes with vertical averaging. We have

Theorem 3.2. The operator $\tilde{\mathbf{B}}$ commutes with vertical averaging. More precisely,

$$
\overline{\tilde{\mathbf{B}}(\mathbf{w}, \mathbf{w})}=\tilde{\mathbf{B}}(\overline{\mathbf{w}}, \overline{\mathbf{w}})=\mathbf{B}_{2 D}(\overline{\mathbf{w}}, \overline{\mathbf{w}})=-\mathbf{P}(\overline{\mathbf{w}} \cdot \nabla \overline{\mathbf{w}}) .
$$


Proof. Let $\mathbf{w}=\overline{\mathbf{w}}+\mathbf{w}^{\perp}$ where the orthogonal field $\mathbf{w}^{\perp}$ verifies $\overline{\mathbf{w}}^{\perp}=0$. Clearly,

$$
\overline{\tilde{\mathbf{B}}(\mathbf{w}, \mathbf{w})}=\tilde{\mathbf{B}}(\overline{\mathbf{w}}, \overline{\mathbf{w}})+\overline{\tilde{\mathbf{B}}\left(\mathbf{w}^{\perp}, \mathbf{w}^{\perp}\right)}
$$

since $\overline{\mathbf{w}}^{\perp}=0$. Thus, the theorem will be proven if we show that

$$
\overline{\tilde{\mathbf{B}}\left(\mathbf{w}^{\perp}, \mathbf{w}^{\perp}\right)}=0 .
$$

The proof actually uses skew-symmetry properties of the 2-waves resonant operator $\mathbf{B}_{\text {II }}$, a corollary from the general skew-symmetry of $\tilde{\mathbf{B}}$ is Lemma 3.1. Let $\overline{\mathbf{H}}_{\sigma}^{1}$ be the restriction of the Hilbert space $\mathbf{H}_{\sigma}^{1}$ to $\operatorname{ker}(\mathbf{P J P})$

$$
\mathbf{H}_{\sigma}^{1}=\overline{\mathbf{H}}_{\sigma}^{1}+\left(\mathbf{H}_{\sigma}^{1}\right)^{\perp} .
$$

Let $\overline{\mathbf{u}}$ be any element in $\overline{\mathbf{H}}_{\sigma}^{1}, \mathbf{w}^{\perp}$ any element in $\left(\mathbf{H}_{\sigma}^{1}\right)^{\perp}$. To prove that $\overline{\tilde{\mathbf{B}}\left(\mathbf{w}^{\perp}, \mathbf{w}^{\perp}\right)}=$ 0 , it suffices to show that

$$
\left.\overline{\left(\tilde{\mathbf{B}}\left(\mathbf{w}^{\perp}, \mathbf{w}^{\perp}\right)\right.}, \overline{\mathbf{u}}\right)=0
$$

for any $\overline{\mathbf{u}}, \mathbf{w}^{\perp}$. Now with $($,$) denoting the complex inner product in L^{2}$, using $k_{3}=-m_{3}, \pm k_{3} / \lambda_{k} \pm m_{3} / \lambda_{m}=0,\left|\lambda_{k}\right|=\left|\lambda_{m}\right|$, hence $\beta_{k}=\beta_{m}$, we obtain:

$$
\begin{aligned}
& \left(\overline{\tilde{\mathbf{B}}\left(\mathbf{w}^{\perp}, \mathbf{w}^{\perp}\right)}, \overline{\mathbf{u}}\right)=\sum_{k, m, k_{3}+m_{3}=0, \beta_{k}=\beta_{m}}\left(w_{k} \operatorname{curl} \Phi_{k} \times w_{m} \Phi_{m}, \overline{\mathbf{u}}\right)= \\
& \sum_{k, n, k_{3}=n_{3}, \beta_{k}=\beta_{n}}\left(w_{k} \operatorname{curl} \Phi_{k} \times w_{-n} \Phi_{-n}, \overline{\mathbf{u}}\right)= \\
& -\sum_{k, m, k_{3}=n_{3}, \beta_{k}=\beta_{n}}\left(w_{k} \operatorname{curl} \Phi_{k} \times \overline{\mathbf{u}}, w_{n} \Phi_{n}\right),
\end{aligned}
$$

where we set $m=-n$, note that $\beta_{-n}=\beta_{n}$, and use $w_{n}=\left(w_{-n}\right)^{*}, \Phi_{n}=\left(\Phi_{-n}\right)^{*}$,* denoting complex conjugate. Here the short hand notation $-n$ has been introduced at the end of Section 2. It then suffices to prove that the last term is identically null for every $\overline{\mathbf{u}}$; this term actually involves a component of $\mathbf{B}_{\mathrm{II}}\left(\overline{\mathbf{w}}, \mathbf{w}^{\perp}\right)$. Apply the skew-symmetry Lemma 3.1 to $\mathbf{u} \equiv \overline{\mathbf{u}}, \mathbf{u}^{\perp}=0, \mathbf{v} \equiv \mathbf{v}^{\perp}, \mathbf{w} \equiv \mathbf{w}^{\perp}, \overline{\mathbf{v}}=\overline{\mathbf{w}}=0$, to obtain:

$$
\begin{aligned}
& \left(\mathbf{B}_{\mathrm{II}}\left(\overline{\mathbf{u}}, \mathbf{v}^{\perp}\right), \mathbf{w}^{\perp}\right)+\left(\mathbf{B}_{\mathrm{III}}\left(\mathbf{v}^{\perp}, \mathbf{v}^{\perp}\right), \mathbf{w}^{\perp}\right)= \\
& -\left(\mathbf{B}_{\mathrm{II}}\left(\overline{\mathbf{u}}, \mathbf{w}^{\perp}\right), \mathbf{v}^{\perp}\right)-\left(\mathbf{B}_{\mathrm{III}}\left(\mathbf{v}^{\perp}, \mathbf{w}^{\perp}\right), \mathbf{v}^{\perp}\right) .
\end{aligned}
$$

Set $\mathbf{v}^{\perp}=\mathbf{w}^{\perp}$ in the above. From the expression for $\mathbf{B}_{\mathrm{III}}\left(\mathbf{w}^{\perp}, \mathbf{w}^{\perp}\right)$ in $(3.20)$ and the skew-symmetry of the triple vector product, it can easily be proven that $\left.\left.\mathbf{B}_{\mathrm{III}}\left(\mathbf{w}^{\perp}, \mathbf{w}^{\perp}\right), \mathbf{w}^{\perp}\right)\right)=0$ for every $\mathbf{w}^{\perp}$ in $\left(\mathbf{H}_{\sigma}^{1}\right)^{\perp}$. Now, from the representation of $\mathbf{B}_{\mathrm{II}}$ in (3.19):

$$
\begin{gathered}
\left(\mathbf{B}_{\mathrm{II}}\left(\overline{\mathbf{u}}, \mathbf{w}^{\perp}\right), \mathbf{w}^{\perp}\right)=\sum_{m, n, m_{3}=n_{3}, \beta_{m}=\beta_{n}}\left(\operatorname{curl} \overline{\mathbf{u}} \times w_{m} \Phi_{m}, w_{n} \Phi_{n}\right)+ \\
\sum_{k, n, k_{3}=n_{3}, \beta_{k}=\beta_{n}}\left(w_{k} \operatorname{curl} \Phi_{k} \times \overline{\mathbf{u}}, w_{n} \Phi_{n}\right) .
\end{gathered}
$$


We now show that the first term on the right hand side of the previous equation is null:

$$
\begin{gathered}
\sum_{m, n, m_{3}=n_{3}, \beta_{m}=\beta_{n}}\left(\operatorname{curl} \overline{\mathbf{u}} \times w_{m} \Phi_{m}, w_{n} \Phi_{n}\right)= \\
-\sum_{m, n, m_{3}=n_{3}, \beta_{m}=\beta_{n}}\left(\operatorname{curl} \overline{\mathbf{u}} \times w_{n}^{*} \Phi_{n}^{*}, w_{m}^{*} \Phi_{m}^{*}\right)= \\
-\sum_{m, n, m_{3}=n_{3}, \beta_{m}=\beta_{n}}\left(\operatorname{curl} \overline{\mathbf{u}} \times w_{-n} \Phi_{-n}, w_{-m} \Phi_{-m}\right),
\end{gathered}
$$

and relabeling $-n=m,-m=n$ (with the short hand notations introduced at the end of Section 2), using $\beta_{-m}=\beta_{m}, \beta_{-n}=\beta_{n}$,

$$
=-\sum_{m, n, m_{3}=n_{3}, \beta_{m}=\beta_{n}}\left(\operatorname{curl} \overline{\mathbf{u}} \times w_{m} \Phi_{m}, w_{n} \Phi_{n}\right)=0 .
$$

Since $\left.\mathbf{B}_{\mathrm{II}}\left(\overline{\mathbf{u}}, \mathbf{w}^{\perp}\right), \mathbf{w}^{\perp}\right)=0$, it now follows from (3.29)

$$
\sum_{k, n, k_{3}=n_{3}, \beta_{k}=\beta_{n}}\left(w_{k} \operatorname{curl} l \Phi_{k} \times \overline{\mathbf{u}}, w_{n} \Phi_{n}\right)=0,
$$

for every $\mathbf{w}^{\perp}$ in $\left(\mathbf{H}_{\sigma}^{1}\right)^{\perp}$. From (3.27) we conclude that

$$
\left.\overline{\left(\tilde{\mathbf{B}}\left(\mathbf{w}^{\perp}, \mathbf{w}^{\perp}\right)\right.}, \overline{\mathbf{u}}\right)=0,
$$

for every $\overline{\mathbf{u}}$ and every $\mathbf{w}^{\perp}$, and $\overline{\tilde{\mathbf{B}}\left(\mathbf{w}^{\perp}, \mathbf{w}^{\perp}\right)}=0$ for every $\mathbf{w}^{\perp}$. $\square$

The above proof only uses the intrinsic algebraic properties of the resonant operators $\tilde{\mathbf{B}}(\mathbf{w}, \mathbf{w}), \tilde{\mathbf{B}}(\mathbf{w}, \mathbf{w})^{\perp}$ and avoids using the specific formulas for the complex eigenfunctions $\Phi_{n}$, including distinguishing between the eigenvalues $\pm \lambda_{m_{1} m_{2} m_{3}}$.

3.2. Strict 3-wave resonances. In this section we show that for all values of $\alpha$, except a countable set, the resonant sets lie in $\left\{k_{3} m_{3} n_{3}=0\right\}$. This is generic case of no strict 3 -wave resonances.

In the case of strict 3 -wave resonances we have $k_{3} m_{3} n_{3} \neq 0$ and the resonant equations become

$$
\pm \frac{1}{\sqrt{\frac{\beta_{k_{1}}^{2}\left(k_{2}, k_{3} \alpha\right)}{k_{3}^{2} \alpha^{2}}+1}} \pm \frac{1}{\sqrt{\frac{\beta_{m_{1}}^{2}\left(m_{2}, m_{3} \alpha\right)}{m_{3}^{2} \alpha^{2}}+1}} \pm \frac{1}{\sqrt{\frac{\beta_{n_{1}}^{2}\left(n_{2}, n_{3} \alpha\right)}{n_{3}^{2} \alpha^{2}}+1}}=0 .
$$

We also have convolutions in the azimuthal $\phi$ and the axial $z$ directions implying $n_{3}=k_{3}+m_{3}, n_{2}=k_{2}+m_{2}$. We recall that for every pair of integers $k_{2}$ and $k_{3}$ the quantities $\beta_{k_{1}}\left(k_{2}, k_{3} \alpha\right)$ are found from the equation

$$
\beta J_{k_{2}}{ }^{\prime}(\beta) \pm k_{2} J_{k_{2}}(\beta) \sqrt{\frac{\beta_{k_{1}}^{2}\left(k_{2}, k_{3} \alpha\right)}{k_{3}^{2} \alpha^{2}}+1}=0 .
$$

For every pair of integers $k_{2}$ and $k_{3}$, Eqs. (3.35) have a countable number of solutions denoted by $\beta\left(k_{1}, k_{2}, k_{3} \alpha\right) ; k_{1}=1,2,3 \ldots$. Similarly, for $\beta\left(m_{1}, m_{2}, m_{3} \alpha\right)$ and $\beta\left(n_{1}, n_{2}, n_{3} \alpha\right)$. 
Eqs. (3.34) can be written in the form

$$
\pm \frac{1}{\sqrt{X}} \pm \frac{1}{\sqrt{Y}} \pm \frac{1}{\sqrt{Z}}=0
$$

where

$$
\frac{\beta^{2}\left(k_{1}, k_{2}, k_{3} \alpha\right)}{k_{3}^{2} \alpha^{2}}+1=X \leftrightarrow\left(\frac{\beta J_{k_{2}}{ }^{\prime}(\beta)}{k_{2} J_{k_{2}}(\beta)}\right)^{2}=X,
$$

with similar expressions for $Y$ and $Z$.

Substituting Eqs. (3.35) in Eqs. (3.34) we obtain

$$
\begin{aligned}
& \pm \frac{k_{2} J_{k_{2}}\left(\beta\left(k_{1}, k_{2}, k_{3} \alpha\right)\right)}{\beta\left(k_{1}, k_{2}, k_{3} \alpha\right) J_{k_{2}}{ }^{\prime}\left(\beta\left(k_{1}, k_{2}, k_{3} \alpha\right)\right)} \pm \frac{m_{2} J_{m_{2}}\left(\beta\left(m_{1}, m_{2}, m_{3} \alpha\right)\right)}{\beta\left(m_{1}, m_{2}, m_{3} \alpha\right) J_{m_{2}}{ }^{\prime}\left(\beta\left(m_{1}, m_{2}, m_{3} \alpha\right)\right)} \\
& \pm \frac{n_{2} J_{n_{2}}\left(\beta\left(n_{1}, n_{2}, n_{3} \alpha\right)\right)}{\beta\left(n_{1}, n_{2}, n_{3} \alpha\right) J_{n_{2}}{ }^{\prime}\left(\beta\left(n_{1}, n_{2}, n_{3} \alpha\right)\right)}=0 .
\end{aligned}
$$

In Eqs. (3.38) $k_{2}, m_{2}, n_{2}, k_{3}, m_{3}, n_{3} \in \mathbf{Z}$ and $k_{1}, m_{1}, n_{1}=1,2,3, \ldots$ Also, $n_{2}=k_{2}+m_{2}$ and $n_{3}=k_{3}+m_{3}$. In fact, we can think of Eqs. (3.38) as a countable set of nonlinear equations for $\alpha$. Clearly, for every fixed $k_{j}, m_{j}, n_{j}$ Eq. (3.38) has at most a countable number of solutions $\alpha$. Thus, we have a countable number of equations and each equation has at most a countable number of solutions $\alpha$. Therefore, the set of parameters $\alpha$ 's for which strict 3-wave resonances can occur is countable and discrete.

Proposition 3.3. The set $\mathcal{K}^{*}$ of parameters $\alpha$ 's for which strict 3-wave resonances can occur is countable and discrete.

3.3. Regularity of fast singular oscillating limit equations. In the generic case of no strict 3-wave resonances $\mathbf{B}_{\mathrm{III}}=0$ and the limit Euler equations (1.35) become

$$
\partial_{t} \mathbf{w}^{\perp}=\mathbf{B}_{\mathrm{II}}\left(\overline{\mathbf{w}}(t), \mathbf{w}^{\perp}\right),\left.\mathbf{w}^{\perp}\right|_{t=0}=\mathbf{w}^{\perp}(0)=\mathbf{U}^{\perp}(0)=\mathbf{U}(0)-\overline{\mathbf{U}}(0) .
$$

where $\overline{\mathbf{w}}(t)$ satisfies $2 \mathrm{D}$ Euler equations with vertically averaged initial data $\left.\overline{\mathbf{w}}\right|_{t=0}$ $=\overline{\mathbf{w}}(0)=\overline{\mathbf{U}}(0)$. Eqs. (3.39) for $\mathbf{w}^{\perp}(t)$ are solved with periodic boundary conditions in the third coordinate and $\left.\mathbf{w}^{\perp} \cdot \mathbf{N}\right|_{\Gamma}=0$. We also have $\left.\operatorname{curl} \mathbf{w}^{\perp} \cdot \mathbf{N}\right|_{\Gamma}=0$.

Eqs, (3.39) possess new 3D conservation laws:

Theorem 3.4. Let $\overline{\mathbf{w}}(t)$ be a solution of 2D-3C Euler Eqs. (1.34). Then for every $\mathbf{w}^{\perp}(t)$ solution of Eqs. (3.39) with initial data $\mathbf{w}^{\perp}(0)$ we have:

$$
\left\|\partial_{3} \mathbf{w}^{\perp}(t)\right\|^{2}=\left\|\partial_{3} \mathbf{w}^{\perp}(0)\right\|^{2},
$$

where $\partial_{3}$ denotes the partial derivative with respect to $x_{3}$.

Proof. Applying $\partial_{3}$ to Eqs. (3.39) and using skew-symmetry property

$$
\left(\mathbf{B}_{\text {II }}\left(\overline{\mathbf{w}}, \partial_{3} \mathbf{w}^{\perp}\right), \partial_{3} \mathbf{w}^{\perp}\right)=0
$$

we obtain

$$
\frac{d}{d t}\left\|\partial_{3} \mathbf{w}^{\perp}\right\|^{2}=0
$$


Moreover, for the initial data and solutions in the function space $\mathbf{H}_{\nu}^{s}$, we have further conservation laws:

THEOREM 3.5. Let $\mathbf{w}^{\perp}(t)$ be solutions of the limit equations (3.39) in the space $\mathbf{H}_{\nu}^{s}$ defined in (3.11). We have, for $0 \leq j \leq s, j$ even:

$$
\left\|\operatorname{curl}^{j} \mathbf{w}^{\perp}(t)\right\|^{2}=\left\|\operatorname{curl}^{j} \mathbf{w}^{\perp}(0)\right\|^{2} .
$$

Proof. Proceed as in the proof of Theorem 3.2, but with $k_{3}=n_{3}$ (and $m_{3}=0$ ) or with $m_{3}=n_{3}\left(\right.$ and $\left.k_{3}=0\right)$, together with $\lambda_{k}^{2}=\lambda_{n}^{2}, \beta_{k}^{2}=\beta_{n}^{2}\left(\right.$ resp. $\lambda_{m}^{2}=\lambda_{n}^{2}, \beta_{m}^{2}=$ $\left.\beta_{n}^{2}\right)$. We have

$$
\begin{aligned}
\left(\operatorname{curl}^{j} \mathbf{B}_{\mathrm{II}}\left(\overline{\mathbf{u}}, \mathbf{w}^{\perp}\right), \operatorname{curl}^{j} \mathbf{w}^{\perp}\right)= & \sum_{m, n, m_{3}=n_{3}, \beta_{m}=\beta_{n}}\left(\operatorname{curl} \overline{\mathbf{u}} \times w_{m}^{\perp} \Phi_{m}, \lambda_{n}^{2 j} w_{n}^{\perp} \Phi_{n}\right)+ \\
& \sum_{k, n, k_{3}=n_{3}, \beta_{k}=\beta_{n}}\left(w_{k}^{\perp} \operatorname{curl} \Phi_{k} \times \overline{\mathbf{u}}, \lambda_{n}^{2 j} w_{n}^{\perp} \Phi_{n}\right) .
\end{aligned}
$$

Consider the second term on the right hand side of (3.43):

$$
\begin{aligned}
\sum_{k, n, k_{3}=n_{3}, \beta_{k}=\beta_{n}}\left(w_{k}^{\perp} \operatorname{curl} \Phi_{k} \times \overline{\mathbf{u}}, \lambda_{n}^{2 j} w_{n}^{\perp} \Phi_{n}\right) & = \\
-\sum_{k, n, k_{3}+n_{3}=0, \beta_{k}=\beta_{n}}\left(w_{k}^{\perp} \operatorname{curl} \Phi_{k} \times \lambda_{n}^{2 j} w_{n}^{\perp} \Phi_{n}, \overline{\mathbf{u}}\right) & = \\
-\sum_{k, n, k_{3}+n_{3}=0, \beta_{k}=\beta_{n}}\left(w_{k}^{\perp} \operatorname{curlcurl}^{j} \Phi_{k} \times w_{n}^{\perp} \operatorname{curl}^{j} \Phi_{n}, \overline{\mathbf{u}}\right) & = \\
-\left(\overline{\tilde{\mathbf{B}}\left(\text { curl }^{j} \mathbf{w}^{\perp}, \text { curl }^{j} \mathbf{w}^{\perp}\right)}, \overline{\mathbf{u}}\right) & =0 ;
\end{aligned}
$$

where we have relabeled $n \rightarrow-n, w_{-n}^{\perp} \rightarrow w_{n}^{\perp}, \Phi_{-n} \rightarrow \Phi_{n}$, and used in an essential way $\lambda_{k}^{j}=\lambda_{n}^{j}$ for $j$ - even). From the operator splitting theorem 3.2 follows the nullity of the last term above. The nullity of the first term on the right hand side of (3.43) is proven in a similar manner.

Note that expansion along the eigenfunctions of curl and PJP requires their completeness at least in $\mathbf{H}_{\nu}^{1}$, cf. Remark 3.1. $\mathrm{q}$

Note that 2D-3C Euler equations only admit conservation of energy and enstrophy. The above conservation laws (3.40)-(3.42) ensure global regularity of the limit Euler equations (3.39).

Theorem 3.6. Let $h / R \notin \mathcal{K}^{*}$. Let $\|\mathbf{w}(0)\|_{\mathbf{H}_{\nu}^{s}} \leq M_{s}, s \geq 1$. Let $T_{1}>0$ fixed, arbitrary large. Then there exists a unique regular solution $\mathbf{w}(t)$ of the limit resonant $3 D$ Euler equations (3.6)-(3.7), for $0 \leq t \leq T_{1}$ :

$$
\|\mathbf{w}(t)\|_{\mathbf{H}_{\nu}^{s}} \leq \tilde{M}_{s}\left(h / R, M_{s}, T_{1}\right) .
$$

4. Long time regularity for finite large $\Omega$. Two major obstacles in extending the fast singular oscillating limit methods developed in [3]-[5] from the periodic lattice case to the cylinder (as well as other axisymmetric domains) are that: (i) PJP is not skew-symmetric with respect to the inner product of classical Sobolev spaces $\mathbf{H}_{\sigma}^{s}(\mathbf{C}), s \geq 1$; (ii) $\mathbf{E}(\Omega t)$ is not an isometry in these spaces ( $\nabla$ does not commute with PJP and $\mathbf{E}(\Omega t)$ ). Item (i) implies that a priori estimates of Eqs. (1.11)-(1.12) in 
Sobolev spaces are $1 / \epsilon=\Omega$ dependent; and (ii) that estimates for $\mathbf{u}(t, x)$, the Poincaré slow variable (van der Pol transformation of $\mathbf{U}(t, x)$, Eq.(3.1)) are not invariant for the physical variable $\mathbf{U}(t, x)$. That invariance was used in an essential way in the convergence proofs of [3]-[5] (periodic case). The resolution of the above requires the introduction of Hilbert spaces with the metric based on the operator curl-norms, with norms equivalent to that of $\mathbf{H}_{\sigma}^{s}(\mathbf{C}), s$ integer, $s \geq 1$.

As before (cf. Eq. 3.11), we restrict ourselves to initial data and solutions in the spaces $(s \geq 3)$ :

$$
\mathbf{H}_{\nu}^{s}(\mathbf{C})=\mathbf{J}^{0} \cap \mathbf{H}_{\sigma}^{s} \cap \operatorname{curl}^{-s}\left(\mathbf{J}^{0}\right),
$$

such that $\mathbf{v} \in \mathbf{H}_{\nu}^{s}$ implies $\operatorname{curl}^{j} \mathbf{v} \cdot \mathbf{N}=0$ on $\Gamma, 0 \leq j \leq s$ More general functional spaces dense in $\mathbf{H}_{\sigma}^{1}$ will be treated in [6].

Lemma 4.1. Let $\mathbf{v} \in \mathbf{H}_{\nu}^{s}, s \geq 1$. Then there exist constants $C_{1}, C_{2}>0$ such that:

$$
C_{1}\|\mathbf{v}\|_{\mathbf{H}_{\sigma}^{s}} \leq\|\mathbf{v}\|_{\mathbf{H}_{\nu}^{s}} \leq C_{2}\|\mathbf{v}\|_{\mathbf{H}_{\sigma}^{s}},
$$

where

$$
\|\mathbf{v}\|_{\mathbf{H}_{\nu}^{s}}^{2}=\left\|\operatorname{curl}^{s} \mathbf{v}\right\|_{L_{2}}^{2}
$$

Proof. Iterated applications of Theorem 2.7 and 2.8, we have equivalence of the "curl-norms" with the usual Sobolev space norms.

From now on, we designate by $\|\mathbf{v}\|_{s}$ the curl-norm of $\mathbf{v}$ defined in Eq. 4.3, and by $<\mathbf{u}, \mathbf{v}\rangle_{s}$ the corresponding inner product of $\mathbf{u}, \mathbf{v}$ in $\mathbf{H}_{\nu}^{s}$. We have the:

Lemma 4.2. Let $\mathbf{u}, \mathbf{v} \in \mathbf{H}_{\nu}^{s}, s \geq 0$; then we have skew-symmetry in the curlnorms:

$$
<\mathbf{P J P u}, \mathbf{v}>_{s}=-<\mathbf{u}, \mathbf{P J P v}>_{s}
$$

and

$$
<\mathbf{P J P u}, \mathbf{u}>_{s}=0
$$

Proof. Obvious for $s=0$; we outline the case $s=1$ :

$$
\begin{aligned}
(\operatorname{curl} \mathbf{P J P} \mathbf{u}, \operatorname{curl} \mathbf{v})_{L_{2}} & =\left(-\frac{\partial \mathbf{u}}{\partial z}, \operatorname{curl} \mathbf{v}\right)_{L_{2}}=\left(\mathbf{u}, \operatorname{curl} \frac{\partial \mathbf{v}}{\partial z}\right)_{L_{2}} \\
& =\left(\operatorname{curl} \mathbf{u}, \frac{\partial \mathbf{v}}{\partial z}\right)_{L_{2}}=-(\operatorname{curl} \mathbf{u}, \operatorname{curl} \mathbf{P J P} \mathbf{v})_{L_{2}},
\end{aligned}
$$

since both $\mathbf{u}, \mathbf{v}, \frac{\partial \mathbf{u}}{\partial z}, \frac{\partial \mathbf{v}}{\partial z}$ satisfies the conditions of Lemma 2.4. The cases $s>1$ follows a similar proof.

The all important Lemmas 4.1, 4.2 allow for local estimates of solutions to the 3D Euler equations (1.11)-(1.12) which are $1 / \epsilon=\Omega$ independent.

Corollary 4.3. The Poincaré-Sobolev unitary operator $E(\Omega t)$ is an isometry on the curl spaces $\mathbf{H}_{\nu}^{s}, s \geq 0$; in particular,

$$
\|\mathbf{U}\|_{s}=\|\mathbf{u}\|_{s}
$$


where $\mathbf{U}(t)=\mathbf{E}(-\Omega t) \mathbf{u}(t)$.

Corollary 4.4. For every $\mathbf{U}$, every $\mathbf{V}$ in the curl spaces $\mathbf{H}_{\nu}^{s}$, we have

$$
\begin{array}{r}
\|\mathbf{B}(\mathbf{U}, \mathbf{V})\|_{s} \leq\|\mathbf{U}\|_{s}\|\mathbf{V}\|_{s+1}, \quad s \geq 2 \\
\left|(\mathbf{B}(\mathbf{U}, \mathbf{V}), \mathbf{V})_{s}\right| \leq\|\mathbf{U}\|_{s}\|\mathbf{V}\|_{s}^{2}, s \geq 3 \\
\left|(\mathbf{B}(\mathbf{U}, \mathbf{V}), \mathbf{V})_{2}\right| \leq\|\mathbf{U}\|_{3}\|\mathbf{V}\|_{2}^{2},
\end{array}
$$

and the same estimates hold for $\mathbf{B}(\Omega t, \mathbf{u}, \mathbf{v}), \tilde{\mathbf{B}}(\mathbf{u}, \mathbf{v})$.

Proof. These are exactly Kato's estimates in classical Sobolev spaces [19]. The estimates in the curl spaces $\mathbf{H}_{\nu}^{s}$ immediately follow from Lemma 4.1 and Corollary 4.3 .

To establish long time regularity of the 3D Euler equations Eqs. (1.20)-(1.21) on $0 \leq t \leq T_{M}, T_{M}$ fixed, arbitrary large, we first establish convergence in $\mathbf{H}_{\nu}^{s}$ (as $\Omega \rightarrow \infty)$ of the solution to that of the limit resonant equations (1.34)-(3.39) on the interval $\left[0, T_{s}\right]$, where $T_{s}$ is some local time of existence of (1.20)-(1.21). We only consider the case of "catalytic resonances", $h / R \notin \mathcal{K}^{*}$. With the help of the long time existence of solutions to the limit resonant equations on $\left[0, T_{M}\right]$, cf. Theorem 3.6, we extend local regularity on $\left[0, T_{s}\right]$ to long-time regularity on $\left[0, T_{M}\right]$ by partitioning $\left[0, T_{M}\right]$ into subintervals of length $T_{s}$ and bootstrapping estimates.

Theorem 4.5. Let $\mathbf{U}(0) \in \mathbf{H}_{\nu}^{\beta}, \beta \geq 3$ and $\|\mathbf{U}(0)\|_{\beta} \leq M_{0 \beta}$, where the $\beta$-norm is the curl-norm (4.3). Then:

(i) there exists $T_{\beta}>0$ such that there exists a unique regular solution of the $3 D$ Euler equations on $0 \leq t \leq T_{\beta}$ which satisfies

$$
\|\mathbf{U}(t)\|_{\beta}^{2} \leq M_{\beta}^{2}, \quad 0 \leq t \leq T_{\beta} ;
$$

moreover $M_{\beta}, T_{\beta}$ do not depend on $\Omega$, but only on $M_{0 \beta}, h / R$.

(ii) For every $\alpha$ such that $\beta \geq \alpha \geq 3$, there exists a constant $C(\beta)$ such that

$$
\|\mathbf{U}(t)\|_{\beta}^{2} \leq\left(\|\mathbf{U}(0)\|_{\beta}^{2}\right) \exp \left(\left(C(\beta) \int_{0}^{T_{\beta}}\|\mathbf{V}(\tau)\|_{\alpha} d \tau\right)+T_{\beta}\right),
$$

where $\alpha$ can be fixed independently of $\beta$.

Proof. (i) is proven by a straightforward adaptation of the proof of Kato [19] in $\mathbf{R}^{3}$ to the cylinder $\mathbf{C}$, replacing the usual Sobolev spaces by the spaces $\mathbf{H}_{\nu}^{\beta}$. Kato's method is a vanishing viscosity limit via local existence for the Navier-Stokes equations (the latter via fixed-point construction, not a Galerkin approximation). That estimates are uniform in $\Omega$ stems from Lemma 4.1 and 4.2. (ii) can then be derived exactly as in Theorem 4.1 of [3], replacing Fourier methods by curl eigenvector function expansions.

We now proceed to estimate the error between solutions $\mathbf{u}(t)$ of Eqs. (3.4)-(3.5) with finite large $\Omega$

$$
\begin{gathered}
\partial_{t} \mathbf{u}=\mathbf{B}(\Omega t, \mathbf{u}, \mathbf{u}), \\
\mathbf{B}(\Omega t, \mathbf{u}, \mathbf{u})=\mathbf{E}(\Omega t) \mathbf{B}(\mathbf{E}(-\Omega t) \mathbf{u}, \mathbf{E}(-\Omega t) \mathbf{u})=\tilde{\mathbf{B}}(\mathbf{u}, \mathbf{u})+\mathbf{B}^{o s c}(\Omega t, \mathbf{u}, \mathbf{u})
\end{gathered}
$$

and solutions $\mathbf{w}(t)$ of the limit resonant 3D Navier-Stokes equations

$$
\begin{aligned}
\partial_{t} \mathbf{w} & =\tilde{\mathbf{B}}(\mathbf{w}, \mathbf{w}), \\
\left.\mathbf{w}\right|_{t=0} & =\mathbf{w}(0)=\mathbf{U}(0)=\mathbf{u}(0) .
\end{aligned}
$$


Let

$$
\mathbf{r}(t)=\mathbf{u}(t)-\mathbf{w}(t), \mathbf{r}(0)=0 .
$$

Recall that $\mathbf{E}(\Omega t)$ is an isometry on the curl spaces $\mathbf{H}_{\nu}^{s}$ spaces. Therefore, estimates for norms of $\mathbf{r}(t)$ yield estimates for the norms of the error

$$
\mathbf{R}(t)=\mathbf{E}(-\Omega t)(\mathbf{u}(t)-\mathbf{w}(t))=\mathbf{U}(t)-\mathbf{E}(-\Omega t) \mathbf{w}(t) .
$$

The equation for the error $\mathbf{r}(t)$ is

$$
\mathbf{r}(t)=\int_{0}^{t}\left(\tilde{\mathbf{B}}(\mathbf{u}, \mathbf{r})+\tilde{\mathbf{B}}(\mathbf{r}, \mathbf{w})+\mathbf{B}^{o s c}(\Omega s, \mathbf{u}(s), \mathbf{u}(s))\right) d s,
$$

where

$$
\mathbf{B}^{o s c}(\Omega t, \mathbf{u}(t), \mathbf{u}(t))=\mathbf{E}(\Omega t) \mathbf{B}(\mathbf{E}(-\Omega t) \mathbf{u}(t), \mathbf{E}(-\Omega t) \mathbf{u}(t))-\mathbf{B}_{2 D}(\overline{\mathbf{u}}, \overline{\mathbf{u}})-\tilde{\mathbf{B}}(\mathbf{u}, \mathbf{u})^{\perp} .
$$

Note that the expansion of $\mathbf{B}^{\text {osc }}$ along eigenfunctions of curl is exactly the expansion in the right hand side of $(3.14)$, with $\left(\mathbf{B}_{2 D}(\overline{\mathbf{u}}, \overline{\mathbf{u}})+\tilde{\mathbf{B}}(\mathbf{u}, \mathbf{u})^{\perp}\right)$ removed. We have

TheOREm 4.6. Assume that the regular solution $\mathbf{U}(t)$ of Eq. (1.11), (1.20) with initial condition $\|\mathbf{U}(0)\|_{s_{\tilde{N}}} \leq M_{s 0}$ exists on $0 \leq t \leq T_{s}$, for some $T_{s}$ (not necessary small), with $\|\mathbf{U}(t)\|_{s} \leq \tilde{\mathbf{M}}_{s}\left(\mathbf{M}_{s 0}, T_{s}, h / R\right)$. Then under conditions $\alpha \geq 2$, $s-\alpha \geq 2$ we have

$$
\|\mathbf{r}(t)\|_{\alpha} \leq \delta(\Omega), \forall t \in\left[0, T_{s}\right]
$$

where $\delta(\Omega) \rightarrow 0$ as $\Omega \rightarrow+\infty ; T_{s}$ is independent from $\Omega ; \delta(\Omega)$ depends on $M_{s 0}, T_{s}$, $\alpha, s$, and $h / R$.

Recall that PJP is skew-symmetric under inner product in the curl-norm spaces, therefore, local small time existence for (3.4)-(3.5) is independent of $\Omega$.

To prove this convergence result we notice that the first two terms inside the integral on the right hand side of (4.16) are linear in $\mathbf{r}$ and we only need to show that the contribution of $\mathbf{B}^{\text {osc }}$ can be made arbitrary small as $\epsilon=1 / \Omega \rightarrow 0$. From (4.17) and (3.14) note that $\mathbf{B}^{o s c}(\tau / \epsilon, \mathbf{u}(t), \mathbf{u}(t))$ is an almost periodic function of $\tau$ with values in $C^{0}\left(\left[0, T_{s}\right] ; \mathbf{H}_{\nu}^{\alpha}\right)$ since the set $D_{l}(k, m, n)= \pm \sigma_{k} \pm \sigma_{m} \pm \sigma_{n}$ is countable.

To prove Theorem 4.6, we will need the nontrivial Theorem 4.12 for Banach space valued almost periodic functions. We recall some basic facts for such functions [9], [16]:

Definition 4.7. Let $F \in C^{0}(\mathbf{R} ; X)$ where $X$ is a Banach space. $F$ is said to be almost periodic if and only if, given an $\epsilon>0$, there exists a length $L(\epsilon)$ such that each interval of $\mathbf{R}$ of length $L$ contains an almost-period $p$ associated to $\epsilon$ :

$$
\sup _{\tau \in \mathbf{R}}\|F(t+p)-F(t)\|_{\tau} \leq \epsilon .
$$

We denote by $A P(\mathbf{R}, X)$ the set of all such functions. For $\mathbf{R}$ - valued almost periodic functions the mean exists:

Proposition 4.8. For any almost periodic function $F$ with values in the Banach space $X$ there exists the mean value

$$
M\{F\}=\lim _{T \rightarrow+\infty} \frac{1}{T} \int_{T_{0}}^{T_{0}+T} F(\tau) d \tau \in X ;
$$


this limit is independent of $T_{0}$, and the convergence is uniform in $T_{0}$.

To every $F(\tau) \in A P(\mathbf{R}, X)$ is associated a unique formal Fourier series:

$$
F(\tau) \sim \sum_{k=1}^{+\infty} A_{k} \exp \left(i \omega_{k} \tau\right), A_{k} \in X,
$$

where the set of frequencies $\left\{\omega_{k}\right\}$ is a countable set $I_{\omega}$. This stems from

Proposition 4.9. Let $F(\tau) \in A P(X)$. Then the quantity $A(\omega)=$ $M\{F(\tau) \exp (-i \omega \tau)\}$ is different from the null element of $X$ only for a set at most countable values of $\omega, \omega_{k} \in I_{\omega}$.

In (4.21) we define $A_{k}=A\left(\omega_{k}\right)$ for those values of $\omega$ for which $A(\omega) \neq 0$. Every $F(\tau) \in A P(X)$ can be uniformly approximated by trigonometric polynomials.

Proposition 4.10. Let $F(\tau) \in A P(X)$, with the formal Fourier series (4.21). Then there exists a sequence of trigonometric polynomials,

$$
\Pi_{m}(\tau)=\sum_{k=1}^{N(m)} r_{k, m} A_{k} \exp \left(i \omega_{k} t\right), N=N(m),
$$

which converges uniformly to $F$ on the whole real line in the strong topology of $X$. The numbers $r_{k, m}$ are rational, depend on $\omega_{k}$ and $m$, but not on $A_{k}$. Moreover, $0 \leq r_{k, m} \leq 1$ and:

$$
\lim _{m \rightarrow+\infty} r_{k, m}=1 .
$$

The polynomials $\Pi_{m}(\tau)$ are called the Bochner-Fejer polynomials of the function $F(\tau)$. Vice-versa, the property of uniform approximation by a sequence of trigonometric polynomials with a countable set of frequencies ensures that the corresponding $F(\tau) \in A P(X)$.

We specifically consider functions in $A P(X)$ where $X=C^{0}\left([0, T] ; H_{\nu}^{s}\right)$, for some $T>0$.

Theorem 4.11. Let $\alpha \geq 2, \beta \geq \alpha+1, \mathbf{U}(t)$ is defined in Theorem 4.4, $0 \leq t \leq T_{\beta}$. Let $\mathbf{U}(t)=\mathbf{E}(-\Omega t) \mathbf{u}(t)$. Then $\mathbf{B}(\Omega \tau, \mathbf{u}(t), \mathbf{u}(t))=$ $\mathbf{E}(\Omega \tau) \mathbf{B}(\mathbf{E}(-\Omega \tau) \mathbf{u}(t), \mathbf{E}(-\Omega \tau) \mathbf{u}(t))$ is an almost periodic function in $\tau$, with values in $C^{0}\left(\left[0, T_{\beta}\right] ; \mathbf{H}_{\nu}^{\alpha}\right)$.

Proof. From the isometry properties of $\mathbf{E}(\Omega \tau)$ on the spaces $\mathbf{H}_{\nu}^{s}$, and the estimate for the bilinear term, we have

$$
\|\mathbf{B}(\Omega \tau, \mathbf{u}(t), \mathbf{u}(t))\|_{\mathbf{H}_{\nu}^{\alpha}} \leq \text { const }\|\mathbf{U}\|_{\mathbf{H}_{\nu}^{\alpha}}\|\mathbf{U}\|_{\mathbf{H}_{\nu}^{\alpha}+1},
$$

for every $\tau \in \mathbf{R}, 0 \leq t \leq T_{\beta}$. Almost periodicity follows from the countability of the set of frequencies $\pm \frac{k_{3}}{\lambda_{k}} \pm \frac{m_{3}}{\lambda_{m}} \pm \frac{n_{3}}{\lambda_{n}}= \pm \sigma_{k} \pm \sigma_{m} \pm \sigma_{n}$, where $\exp \left( \pm i \sigma_{k} \Omega \tau\right)$ are eigenvalues of $\mathbf{E}( \pm \Omega \tau)_{k}$, and the countability of the eigenvalues $\pm \lambda_{k}$ of the curl operator; the sufficient condition for a function to belong to $A P(X)$ is that it is uniformly approximated by a sequence of trigonometric polynomials with a countable set of frequencies.

To prove the error estimate Theorem 4.6 we need in a crucial way the following general result for almost periodic functions, for which we could not find a proof in the literature. 
TheOREM 4.12. Let $F(t, \tau) \in A P\left(\mathbf{R} ; C_{0}\left(\left[0, T_{0}\right] ; \mathbf{E}\right)\right)$ be almost periodic functions of the variable $\tau$, with values in $C_{0}\left(\left[0, T_{0}\right] ; \mathbf{E}\right), t \in\left[0, T_{0}\right], \mathbf{E}$ is a Hilbert space. Let

$$
F(t, \tau) \sim \sum_{j \in J} F_{j}(t) \exp \left(i \omega_{j} \tau\right)
$$

in the sense of Banach space valued almost periodic functions in $\tau, F_{j}(t) \in$ $C_{0}\left(\left[0, T_{0}\right] ; \mathbf{E}\right)$, over the (countable) set $J$ of frequencies. If

$$
\left.\sup _{\tau \in \mathbf{R}} \sup _{0 \leq t \leq T_{0}}|| F(t, \tau)\right|_{\mathbf{E}} \leq M_{f} \text {, and if }\left|\omega_{j}\right| \geq \eta>0 \text { on } J
$$

and if $F(t, \tau)$ is uniformly Lipschitz in $t$

$$
\left\|F\left(t_{2}, \tau\right)-F\left(t_{1}, \tau\right)\right\|_{\mathbf{E}} \leq L_{F}\left|t_{2}-t_{1}\right|,
$$

the latter uniformly on $\left[0, T_{0}\right]$ and uniformly in $\tau \in \mathbf{R}$, then:

$$
\mathbf{E}-\lim _{\epsilon \rightarrow 0} \int_{0}^{T} F(t, t / \epsilon) d t=0 ;
$$

and the limit also converges uniformly on $0 \leq T \leq T_{0}$.

Proof of Theorem 4.12. Let $F(t, \tau) \sim \sum_{k=1}^{N(m)} A_{k}(t) \exp \left(i \omega_{k} \tau / \epsilon\right)$, where $A_{k}(t) \epsilon$ $C^{0}\left(\left[0, T_{0}\right] ; E\right)$ and

$$
A_{k}(t)=\lim _{T \rightarrow+\infty} \frac{1}{T} \int_{0}^{T} F(t, \tau) \exp \left(-i \omega_{k} \tau\right) d \tau
$$

in the topology of $C^{0}\left(\left[0, T_{0}\right] ; E\right)$. From the above, and the hypothesis of the theorem, we easily deduce

$$
\begin{array}{r}
\sup _{0 \leq t \leq T_{0}}|| A_{k}(t) \|_{E} \leq M_{f} \\
\left\|A_{k}\left(t_{2}\right)-A_{k}\left(t_{1}\right)\right\|_{E} \leq L_{f}\left|t_{2}-t_{1}\right|, \forall t_{1}, t_{2} \in\left[0, T_{0}\right] .
\end{array}
$$

Given some $\eta^{*}$, consider the Bochner-Fejer polynomial $\Pi_{m}$ uniformly approximating $F(t, \tau)$ with the error $\eta^{*}$ in the topology of $C^{0}\left(\left[0, T_{0}\right] ; E\right)$ :

$$
\begin{array}{r}
\Pi_{m}(t, \tau)=\sum_{k=1}^{N(m)} r_{k m} A_{k}(t) \exp \left(i \omega_{k} \tau\right), \\
\sup _{\tau \in \mathbf{R}} \sup _{0 \leq t \leq T_{0}}|| F(t, \tau)-P_{m}(t, \tau) \|_{E} \leq \eta^{*},
\end{array}
$$

in the above, set:

$$
a_{k}(t)=r_{k m} A_{k}(t)
$$

and since $0 \leq r_{k m} \leq 1$, clearly

$$
\begin{array}{r}
\sup _{0 \leq t \leq T_{0}}\left\|a_{k}(t)\right\|_{E} \leq M_{f}, \\
\left\|a_{k}\left(t_{2}\right)-a_{k}\left(t_{1}\right)\right\|_{E} \leq L_{f}\left|t_{2}-t_{1}\right|, \forall t_{1}, t_{2} \in\left[0, T_{0}\right] .
\end{array}
$$

We now proceed to estimate $\int_{0}^{T} F(t, t / \epsilon) d t$; replacing $\tau$ by $t / \epsilon$ on $\Pi_{m}(t, \tau)$, we have:

$$
\left\|\int_{0}^{T} F(t, t / \epsilon) d t\right\|_{E} \leq\left\|\int_{0}^{T} \Pi_{m}(t, t / \epsilon) d t\right\|_{E}+\eta^{*} T
$$


We proceed to estimate the integral of $\Pi_{m}(t, t / \epsilon)$ :

$$
\begin{array}{r}
\int_{0}^{T} \Pi_{m}(t, t / \epsilon) d t=\sum_{k=1}^{N(m)} \int_{0}^{T} a_{k}(t) \exp \left(i \omega_{k} t / \epsilon\right) d t= \\
-\sum_{k=1}^{N} \int_{0}^{T} a_{k}(t) \exp \left(i \omega_{k}\left(t+\frac{\epsilon \pi}{\omega_{k}}\right) / \epsilon\right) d t= \\
\frac{1}{2} \sum_{k=1}^{N}\left(\int_{0}^{T} a_{k}(t) \exp \left(i \omega_{k} t / \epsilon\right) d t-\int_{\frac{\epsilon \pi}{\omega_{k}}}^{T+\frac{\epsilon \pi}{\omega_{k}}} a_{k}\left(t-\frac{\epsilon \pi}{\omega_{k}}\right) \exp \left(i \omega_{k} t / \epsilon\right) d t\right)= \\
\frac{1}{2} \sum_{k=1}^{N}\left(\int_{\frac{\epsilon \pi}{\omega_{k}}}^{T}\left(a_{k}(t)-a_{k}\left(t-\frac{\epsilon \pi}{\omega_{k}}\right)\right) \exp \left(i \omega_{k} t / \epsilon\right) d t+\right. \\
\int_{0}^{\frac{\epsilon \pi}{\omega_{k}}} a_{k}(t) \exp \left(i \omega_{k} t / \epsilon\right) d t+\int_{T}^{T+\frac{\epsilon \pi}{\omega_{k}}} a_{k}\left(t-\frac{\epsilon \pi}{\omega_{k}}\right) \exp \left(i \omega_{k} t\right) d t,
\end{array}
$$

where in the last equation, we use the hypothesis $\frac{1}{\omega_{k}} \leq \frac{1}{\eta}$ on the set $J$ of frequencies, to choose $\epsilon$ small enough to ensure $\frac{\epsilon \pi}{\left|\omega_{k}\right|}<<1$. Using uniform bounds and uniform Lipschitzianity of the $a_{k}(t)$, we obtain:

$$
\left\|\int_{0}^{T} \Pi_{m}(t, t / \epsilon) d t\right\|_{E} \leq \frac{N(m)}{2}\left(\frac{\epsilon \pi}{\left|\omega_{k}\right|} L_{f} T+2 \frac{\epsilon \pi}{\left|\omega_{k}\right|} M_{f}\right) \leq \frac{N(m)}{2} \frac{\epsilon \pi}{\eta} T\left(L_{f}+2 M_{f} / T\right),
$$

and

$$
\left\|\int_{0}^{T} F(t, t / \epsilon) d t\right\|_{E} \leq \frac{N(m)}{2} \frac{\epsilon \pi}{\eta} T\left(L_{f}+2 M_{f} / T\right)+\eta^{*} T .
$$

Given any $\delta$, we first choose $\eta^{*}$ such that $\eta^{*} T_{0}<\delta / 3$, which fixes $m$ and $N(m)$. Then given $T_{0}, \eta, N(m)$, choose $\epsilon$ such that $\frac{\epsilon \pi}{\eta} \frac{N(m)}{2} T_{0} L_{f} \leq \frac{\delta}{3}$, and $\frac{\epsilon \pi}{\eta} N(m) M_{f} \leq \frac{\delta}{3}$.

Proof of Theorem 4.6. The $\mathbf{H}_{\nu}^{\alpha}$-norm of $\mathbf{r}(t)$ satisfies:

$$
\frac{1}{2}\|\mathbf{r}(T)\|_{\alpha}^{2}=\int_{0}^{t}\left((\tilde{\mathbf{B}}(\mathbf{u}, \mathbf{r}), \mathbf{r})_{\alpha}+(\tilde{\mathbf{B}}(\mathbf{r}, \mathbf{w}), \mathbf{r})_{\alpha}+\left(\mathbf{B}^{o s c}(t / \epsilon, \mathbf{u}(t), \mathbf{u}(t)), \mathbf{r}(t)\right)_{\alpha}\right) d t
$$

From Corollary 4.4., with $\alpha \geq 2$ :

$$
\begin{array}{r}
\left|(\tilde{\mathbf{B}}(\mathbf{u}, \mathbf{r}), \mathbf{r})_{\alpha}\right| \leq\|\mathbf{u}\|_{\alpha}\|\mathbf{r}\|_{\alpha}^{2} \text { for } \alpha \geq 3 \\
\leq\|\mathbf{u}\|_{3}\|\mathbf{r}\|_{2}^{2} \text { for } \alpha=2 \\
\left|(\tilde{\mathbf{B}}(\mathbf{r}, \mathbf{w}), \mathbf{r})_{\alpha}\right| \leq\|\mathbf{w}\|_{\alpha+1}\|\mathbf{r}\|_{\alpha}^{2} \text { for } \alpha \geq 2
\end{array}
$$

and since $s$ is chosen $s \geq \alpha+2,\|\mathbf{u}\|_{\alpha} \leq \tilde{\mathbf{M}}_{s},\|\mathbf{w}\|_{\alpha+1} \leq \tilde{\mathbf{M}}_{s}, 0 \leq t \leq T_{s}$.

To apply Theorem 4.12 to $\mathbf{B}^{\text {osc }}(\Omega t, \mathbf{u}(t), \mathbf{u}(t))$ one needs $\left|\omega_{j}\right|=\left|\sigma_{k} \pm \sigma_{m} \pm \sigma_{n}\right|>\eta$ uniformly in $k, m, n$, For this, define $\pi_{R} \mathbf{u}$ (similarly $\pi_{R} \mathbf{w}$ ) the projection of $\mathbf{u}$ onto the curl eigenvector functions with $\left|\lambda_{k}\right|,\left|\lambda_{m}\right|,\left|\lambda_{n}\right| \leq R$, with:

$$
\left\|\mathbf{u}-\pi_{R} \mathbf{u}\right\|_{\mathbf{H}_{\nu}^{\alpha}} \leq \tilde{\mathbf{M}}_{s} R^{\alpha-s}, s>\alpha .
$$


Then $\left| \pm \sigma_{k} \pm \sigma_{m} \pm \sigma_{n}\right|>\eta(R)$ for $\pi_{R} \mathbf{u}$ in Eq. (4.17), which controls the small divisor estimate ([1]). Precisely, $1 /\left|D_{l}(k, m, n)\right| \leq 1 / \eta(R)$, for $k, m, n$ not in the set $K$, where $K$ is the resonance set defined in (3.15), whenever $\left|\lambda_{k}\right|,\left|\lambda_{m}\right|,\left|\lambda_{n}\right| \leq R$.

$\mathbf{B}^{\text {osc }}(\Omega t, \mathbf{u}(t), \mathbf{u}(t))$ is decomposed as

$$
\begin{gathered}
\mathbf{B}^{o s c}(\Omega t, \mathbf{u}, \mathbf{u})=\pi_{R} \mathbf{B}^{o s c}\left(\Omega t, \pi_{R} \mathbf{u}, \pi_{R} \mathbf{u}\right)+\pi_{R} \mathbf{B}^{o s c}\left(\Omega t, \mathbf{u},\left(I-\pi_{R}\right) \mathbf{u}\right)+(4.41) \\
\pi_{R} \mathbf{B}^{o s c}\left(\Omega t,\left(I-\pi_{R}\right) \mathbf{u}, \mathbf{u}\right)+\left(I-\pi_{R}\right) \mathbf{B}^{o s c}(\Omega t, \mathbf{u}, \mathbf{u}) .
\end{gathered}
$$

The contributions of the last three terms on the right hand side of (4.41) to the time integral of $\mathbf{B}^{\text {osc }}$ in (4.38) are estimated with the help of (4.40) and Corollary 4.4:

$$
\begin{gathered}
\left|\left(\pi_{R} \mathbf{B}^{o s c}\left(\Omega t, \mathbf{u},\left(I-\pi_{R}\right) \mathbf{u}\right), \mathbf{r}\right)_{\alpha}\right| \leq\|\mathbf{u}\|_{\alpha} \tilde{\mathbf{M}}_{s} R^{\alpha+1-s}\|\mathbf{r}\|_{\alpha} \leq 2 \tilde{\mathbf{M}}_{s}^{3} R^{\alpha+1-s} \\
\left|\left(\pi_{R} \mathbf{B}^{o s c}\left(\Omega t,\left(I-\pi_{R}\right) \mathbf{u}, \mathbf{u}\right), \mathbf{r}\right)_{\alpha}\right| \leq\|\mathbf{u}\|_{\alpha+1} \tilde{\mathbf{M}}_{s} R^{\alpha-s}\|\mathbf{r}\|_{\alpha} \leq 2 \tilde{\mathbf{M}}_{s}^{3} R^{\alpha-s} \\
\left.\mid\left(\left(I-\pi_{R}\right) \mathbf{B}^{o s c}(\Omega t, \mathbf{u}, \mathbf{u}), \mathbf{r}\right)_{\alpha}\right) \mid \leq\|\mathbf{u}\|_{\alpha}\|\mathbf{u}\|_{\alpha+1}\|\mathbf{r}\|_{s} R^{\alpha-s} \leq 2 \tilde{\mathbf{M}}_{s}^{3} R^{\alpha-s}
\end{gathered}
$$

where we estimate $\|\mathbf{r}\|_{\alpha} \leq\|\mathbf{u}\|_{\alpha}+\|\mathbf{w}\|_{\alpha} \leq 2 \tilde{\mathbf{M}}_{s}$.

Finally apply Theorem 4.12 to the integral

$\int_{0}^{T}\left(\pi_{R} \mathbf{B}^{\text {osc }}\left(t / \epsilon, \pi_{R} \mathbf{u}(t), \pi_{R} \mathbf{u}(t)\right), \mathbf{r}\right)_{\alpha} d t$, with $\mathbf{E}=C^{0}([0, T] ; \mathbf{R})$, and $\epsilon=1 / \Omega$.

Let $F(t, t / \epsilon)$ defined as the $\alpha$-inner product within (4.45). The only technical point left to verify is the uniform Lipschitzianity of $F(t, t / \epsilon)$. This is easily established via the Lipschitz properties of $\mathbf{u}(t), \mathbf{r}(t)=\mathbf{u}(t)-\mathbf{w}(t)$ on $0 \leq t \leq T_{s}$ :

$$
\begin{array}{r}
\left\|\mathbf{u}\left(t_{2}\right)-\mathbf{u}\left(t_{1}\right)\right\|_{\alpha} \leq \tilde{\mathbf{M}}_{s}^{2}\left|t_{2}-t_{1}\right|, \text { for } s \geq \alpha+1, \\
\left\|\mathbf{r}\left(t_{2}\right)-\mathbf{r}\left(t_{1}\right)\right\|_{\alpha} \leq 2 \tilde{\mathbf{M}}_{s}^{2}\left|t_{2}-t_{1}\right|, \text { for } s \geq \alpha+1, \\
\left\|\mathbf{u}\left(t_{2}\right)-\mathbf{u}\left(t_{1}\right)\right\|_{\alpha+1} \leq \tilde{\mathbf{M}}_{s}^{2}\left|t_{2}-t_{1}\right|, \text { provided } s \geq \alpha+2,
\end{array}
$$

and

$$
\left|F\left(t_{2}, \tau / \epsilon\right)-F\left(t_{1}, \tau / \epsilon\right)\right| \leq L_{F}\left|t_{2}-t_{1}\right|
$$

where $L_{F} \leq 5 \tilde{\mathbf{M}}_{s}^{4}\left|t_{2}-t_{1}\right|$, uniformly in $\tau$. To complete the proof, we first choose $R$ large enough so that $2 \tilde{\mathbf{M}}_{s}^{3}\left(R^{\alpha+1-s}+2 R^{\alpha-s}\right)$ is small enough. Once $R$ fixed, we choose $\Omega=1 / \epsilon$ large enough so that the integral $\left|\int_{0}^{T} F(t, t / \epsilon) d t\right|$ is small enough. Conclusion follows from a straightforward Gronwall's lemma applied to

$$
\|\mathbf{r}\|_{\alpha}^{2} \leq \int_{0}^{T} 4 \tilde{\mathbf{M}}_{s}\|\mathbf{r}(\mathbf{t})\|_{\alpha}^{2} d t+\gamma\left(\tilde{\mathbf{M}}_{s}, \Omega\right)
$$

where $\gamma\left(\tilde{\mathbf{M}}_{s}, \Omega\right) \rightarrow 0$ as $\Omega \rightarrow+\infty$.

In Theorem $4.6 \mathbf{u}(t)$ converges strongly to $\mathbf{w}(t)$. The original $\mathbf{U}(t)$ also converges strongly to $\mathbf{E}(-\Omega t) \mathbf{w}(t)$. But $\mathbf{U}(t)$ has no strong limit in $\mathbf{H}_{\nu}^{s}$ as $\Omega \rightarrow \infty$, hence the singular nature of the fast oscillating limit.

From the global regularity Theorem 3.6 for the 3D resonant Euler equations and Theorems 4.5-4.6 we bootstrap long-time regularity for Eqs.(1.11)-(1.12) for $\Omega$ large but finite. 
Proof of Theorem 1.2. We complete in some detail the proof of Theorem 1.2, as the bootstrapping arguments are rather different from the usual classical procedure for Navier-Stokes equations [23]. Let $T_{m}$ fixed, arbitrary large. Let $\left\|\tilde{\mathbf{V}}_{0}(y)\right\|_{s}=$ $\left\|\mathbf{U}_{0}(x)\right\|_{s} \leq M_{s 0}, s \geq 4$. From Theorem 3.6, with $\|\mathbf{w}(0)\|_{\alpha}=\left\|\mathbf{U}_{0}\right\|_{\alpha} \leq M_{\alpha 0} \leq M_{s 0}$, for some fixed $\alpha \geq 3, s-\alpha \geq 1$, there exists $\tilde{M}_{\alpha}$ such that:

$$
\|\mathbf{w}(t)\|_{\alpha} \leq \tilde{\mathbf{M}}_{\alpha}\left(M_{s 0}, T_{M}, h / R\right) \text { on } 0 \leq t \leq T_{m}
$$

for the solution of the resonant 3D Euler equations. We need the:

Corollary 4.13. Let $\left\|\mathbf{U}\left(t_{1}\right)\right\|_{\alpha} \leq R_{\alpha}$ at some $t_{1} \geq 0, \alpha \geq 3, R_{\alpha}>0$ given. Then there exists $T_{\alpha}\left(R_{\alpha}, h / R\right)$ such that

$$
\|\mathbf{U}(t)\|_{\alpha} \leq 4 R_{\alpha} \quad \text { on }\left[t_{1}, t_{1}+T_{\alpha}\right]
$$

moreover if $\mathbf{U}\left(t_{1}\right) \in \mathbf{H}_{\nu}^{s}$ for some $s>\alpha$ :

$$
\begin{aligned}
\|\mathbf{U}(t)\|_{s}^{2} & \leq\left\|\mathbf{U}\left(t_{1}\right)\right\|_{s}^{2} \exp \left\{\left(C(s)\left(\sup _{t_{1} \leq t \leq t_{1}+T_{\alpha}}\|\mathbf{U}\|_{\alpha}\right)+1\right)\left(t-t_{1}\right)\right\} \\
& \leq\left\|\mathbf{U}\left(t_{1}\right)\right\|_{s}^{2} \exp \left\{\left(4 C(s) R_{\alpha}+1\right)\left(t-t_{1}\right)\right\} \quad \text { on }\left[t_{1}, t_{1}+T_{\alpha}\right] .
\end{aligned}
$$

Proof. First, apply Theorem 4.5 with $\alpha=\beta$ to derive Eqs. (4.50). Then apply (ii) in Theorem 4.5 with $\beta=s$.

We now choose, with $\tilde{M}_{\alpha}$ given by Eqs. (4.49):

$$
R_{\alpha}=3 \tilde{M}_{\alpha},
$$

hence $T_{\alpha}=T_{\alpha}\left(M_{s 0}, T_{m}, \alpha, h / R\right)$. Let $Q_{\alpha}=4 C(s) R_{\alpha}+1$. We define:

$$
\tilde{M}_{s}^{2}=M_{s 0}^{2} \exp Q_{\alpha}\left(T_{m}+T_{\alpha}\right),
$$

and we shall demonstrate that:

$$
\|\mathbf{U}(t)\|_{s}^{2} \leq \tilde{M}_{s}^{2} \quad \text { on } 0 \leq t \leq T_{m},
$$

by choosing $\Omega$ large enough to make the error $\delta(\Omega)$ (in Theorem 4.6) uniformly small on the sequence of intervals $\left[0, T_{\alpha}\right],\left[T_{\alpha}, 2 T_{\alpha}\right], \ldots,\left[n T_{\alpha},(n+1) T_{\alpha}\right]$, where $n T_{\alpha} \leq T_{m}<$ $(n+1) T_{\alpha}$. We apply Theorem 4.6 on the global interval $\left[0, T_{m}\right]$, assuming a priori the estimate (4.54) (which will be shown self-consistent under bootstrapping); we choose such large $\Omega$ that $\delta(\Omega)$ is so small and the assertion of Theorem 4.6 holds with $T_{s} \equiv T_{m}$, and for $\Omega \geq \Omega_{1}$, the constant $\delta(\Omega)$ satisfies:

$$
\delta(\Omega) \leq R_{\alpha} / 2 \quad \text { for } \Omega \geq \Omega_{1}\left(\tilde{M}_{s}, T_{m}, \alpha, s, h / R\right) ;
$$

equivalently, $\Omega_{1}$ depends only on $M_{s 0}, T_{m}, \alpha, s, h / R$. Hence, for $0 \leq t \leq T_{m}$ :

$$
\|\mathbf{U}(t)\|_{\alpha} \leq\|\mathbf{U}(t)-\mathbf{E}(-\Omega t) \mathbf{w}(t)\|_{\alpha}+\|\mathbf{w}(t)\|_{\alpha} \leq R_{\alpha} / 2+R_{\alpha} / 3 \leq R_{\alpha} .
$$

We now apply Corollary 4.13 on $\left[0, T_{\alpha}\right]$, with the choice of $R_{\alpha}$ in Eqs. (4.52):

$$
\left\|\mathbf{U}\left(T_{\alpha}\right)\right\|_{s}^{2} \leq M_{s 0}^{2} \exp \left(Q_{\alpha} T_{\alpha}\right) .
$$

From the uniform estimate (4.56) for $\|\mathbf{U}(t)\|_{\alpha}$, valid at $t=T_{\alpha}$, we apply again Corollary 4.13 on $\left[T_{\alpha}, 2 T_{\alpha}\right]$ :

$$
\|\mathbf{U}(t)\|_{s}^{2} \leq M_{s 0}^{2} \exp \left(Q_{\alpha} T_{\alpha}\right) \exp \left(Q_{\alpha} T_{\alpha}\right)
$$


and repeating the argument on the interval $\left[k T_{\alpha},(k+1) T_{\alpha}\right], k \leq n$ :

$$
\|\mathbf{U}(t)\|_{s}^{2} \leq M_{s 0}^{2} \exp \left(k Q_{\alpha} T_{\alpha}\right) \exp \left(Q_{\alpha} T_{\alpha}\right),
$$

since $\left\|\mathbf{U}\left(k T_{\alpha}\right)\right\|_{\alpha} \leq R_{\alpha}$. Completing the bootstrapping, the maximal estimate for $\|\mathbf{U}(t)\|_{s}^{2}$ occurs on $\left[n T_{\alpha}, T_{m}\right]$ :

$$
\begin{aligned}
\|\mathbf{U}(t)\|_{s}^{2} & \leq M_{s 0}^{2} \exp \left(n Q_{\alpha} T_{\alpha}\right) \exp \left(Q_{\alpha}\left(T_{m}-n T_{\alpha}\right)\right) \\
& \leq \tilde{M}_{s}^{2}
\end{aligned}
$$

which corroborates the self-consistency of the choice $\|\mathbf{U}(t)\|_{s} \leq \tilde{M}_{s}$ in the application of Theorem 4.6 for a uniform $\delta(\Omega)$. The proof of Theorem 1.2 is then completed with the canonical transformation (1.17)-(1.18) between $\mathbf{V}(t, y)$ and $\mathbf{U}(t, x)$ and its isometry properties.

The case $h / R \in \mathcal{K}^{*}$ includes the quadratic resonant operator $\mathbf{B}_{I I I}\left(\mathbf{w}^{\perp}, \mathbf{w}^{\perp}\right)$ in Eqs. (1.35). We have not found new conservation laws for the latter besides energy and helicity. A most interesting issue is the possibility of singularity and blow-up for the full resonant Euler equations (1.35). Partial results in the periodic lattice geometry are derived in [5], where (1.35) is demonstrated to be equivalent to a countable sequence of uncoupled finite dimension dynamical systems, in the generic case.

Acknowledgment. We would like to thank Professors V.I. Arnold, C. Foias, A. Fursikov, O.A. Ladyzhenskaya, H.K. Moffatt, G. Papanicolaou and Ya. Sinai for the very useful discussions. Professor V.A. Solonnikov has been most helpful in bringing to our attention the earlier work of his and his colleagues on magnetohydrodynamics. We acknowledge the support of the AFOSR grant F49620-02-10026.

\section{REFERENCES}

[1] V.I. Arnold, Small denominators. I. Mappings of the circumference onto itself, Amer. Math. Soc. Transl. Ser. 2, 46(1965), pp. 213-284.

[2] V.I. Arnold and B.A. Khesin, Topological Methods in Hydrodynamics, Applied Mathematical Sciences, 125(1997), Springer.

[3] A. Babin, A. Mahalov, and B. Nicolaenko, Global regularity and integrability of 3D Euler and Navier-Stokes equations for uniformly rotating fluids, Asymptotic Analysis, 15(1997), pp. 103-150.

[4] A. Babin, A. Mahalov and B. Nicolaenko, Global regularity of 3D rotating Navier-Stokes equations for resonant domains, Indiana Univ. Math. J., 48:3(1999), pp. 1133-1176.

[5] A. Babin, A. Mahalov and B. Nicolaenko, 3D Navier-Stokes and Euler equations with initial data characterized by uniformly large vorticity, Indiana Univ. Math. J., 50(2001), pp. $1-35$.

[6] C. Bardos, F. Golse, A. Mahalov and B. Nicolaenko, Long-time regularity of 3D Euler equations with initial data characterized by uniformly large vorticity in cylindrical domains, (2005), to appear.

[7] J.T. Beale, T. Kato And A. Majda, Remarks on the breakdown of smooth solutions for the $3 D$ Euler equations, Commun. Math. Phys., 94(1984), pp. 61-66.

[8] A. Bensoussan, J.-L. Lions and G. Papanicolaou, Asymptotic Analysis for Periodic Structures, North-Holland Pub. Co., New York, (1978).

[9] A.S. Besicovitch, Almost Periodic Functions, Dover, New York, (1954).

[10] N.N. Bogoliubov And Y. A. Mitropolsky, Asymptotic Methods in the Theory of Non-linear Oscillations, Gordon and Breach Science Publishers, New York, (1961).

[11] J.P. Bourguignon And H. Brezis, Remark on the Euler equations, J. Func. Anal., 15(1974), pp. 341-363.

[12] E.B. Byкhovskil, Solutions of problems of mixed type of the Maxwell's equations for ideally conducting boundaries, Vestnik Leningrad Univ., 13(1957), pp. 50-66. 
[13] E.B. Bykhovskit AND N.V. Smirnov, On orthogonal expansions in spaces of square integrable vector functions and operators of vector analysis, Trudy Math. Inst. V.A. Steklov, Special Volume on Mathematical Problems in Hydrodynamics and Magnetohydrodynamics, 59(1960), pp. 5-36.

[14] E. CARTAN, Sur les petites oscillations d'une masse fluid, Bull. Sci. Math., 46(1922), pp. 317352 and pp. 356-369.

[15] S. Childress, New solutions of the kinematic dynamo problem, J. Math. Phys., 11(1970), pp. 3063-3076.

[16] C. Corduneanu, Almost periodic Functions, Wiley-Interscience, New York, (1968).

[17] G. Duvaut And J.L. Lions, The Inequalities in Mechanics and Physics, Springer-Verlag, (1976).

[18] K. Friedrichs, Differential forms on Riemannian manifolds, Comm. Pure Appl. Math, 8:2(1955)

[19] T. Kato, Nonstationary flows of viscous and ideal fluids in $\mathbf{R}^{\mathbf{3}}$, J. Func. Anal., 9(1972), pp. 296-305.

[20] N.D. Kopachevsky and S.G. Krein, Operator Approach to Linear Problems of Hydrodynamics, Operator Theory: Advances and Applications, 128(2001), Birkhauser Verlag.

[21] O.A. Ladyzhenskaya Ed., Trudy Math. Inst. V.A. Steklov, Special Volume on Mathematical Problems in Hydrodynamics and Magnetohydrodynamics, 59(1960).

[22] O.A. Ladyzhenskaya, On the unique global solvability of the 3D Cauchy problem for the Navier-Stokes equations under conditions of axisymmetry, Boundary Value problems of Math. Phys. and Related Problems No. 2, Zapiski Seminarov LOMI, 7(1968), pp. 155-177.

[23] O.A. Ladyzhenskaya, The Mathematical Theory of Viscous Incompressible Flow, 2nd ed., Gordon and Breach, New York, (1969).

[24] O.A. Ladyzhenskaya and V.A. Solonnikov, Solution of some nonstationary problems of magnetohydrodynamics for viscous incompressible fluids, Trudy Math. Inst. V.A. Steklov, Special Volume on Mathematical Problems in Hydrodynamics and Magnetohydrodynamics, 59(1960), pp. 115-173.

[25] J. Leray, Sur le mouvement d'un liquide visqueux emplissant l'espace, Acta Math., 63(1934), pp. $193-248$.

[26] A. Mahalov, S. Leibovich and E.S. Titi, Invariant helical subspaces for the Navier-Stokes Equations, Arch. for Rational Mech. and Anal., 112:3(1990), pp. 193-222.

[27] A. Mahalov and B. Nicolaenko, Global solvability of the three-dimensional Navier-Stokes equations with uniformly large initial vorticity, Russian Math. Surveys, 58:2(2003), pp. $287-318$.

[28] H. Poincaré, Sur la précession des corps déformables, Bull. Astronomique, 27(1910), pp. 321-356.

[29] J.V. Ralston, On stationary modes in inviscid rotating fluids, J. Math. Anal. and Appl., 44(1973), pp. 366-383.

[30] S. Schоснет, Resonant nonlinear geometric optics for weak solutions of conservation laws, J. Diff. Eq., 113(1994), pp. 473-503.

[31] S. Schochet, Fast singular limits of hyperbolic PDE's, J. Diff. Eq., 114(1994), pp. 476-512.

[32] S. L. Sobolev, Ob odnoi novoi zadache matematicheskoi fiziki, Izvestiia Akademii Nauk SSSR, Ser. Matematicheskaia, 18:1(1954), pp. 3-50.

[33] V.A. Solonnikov, Overdetermined elliptic boundary value problems, Boundary Value problems of Math. Phys. and Related Problems No. 5, Zapiski Seminarov LOMI, 21(1972), pp. 112158.

[34] E.M. Stein, Singular integrals and differentiability properties of functions, Princeton University Press, (1970).

[35] W. Wolibner, Un théorème sur l'existence du mouvement plan d'un fluide parfait, homogéne et incompressible, pendant un temps infiniment long, Mat. Z., 37(1933), pp. 698-726.

[36] V.I. Yudovich, Non stationary flow of an ideal incompressible liquid, Zb. Vych. Mat., 3(1963), pp. $1032-1066$ 
A. MAHALOV ET. AL. 